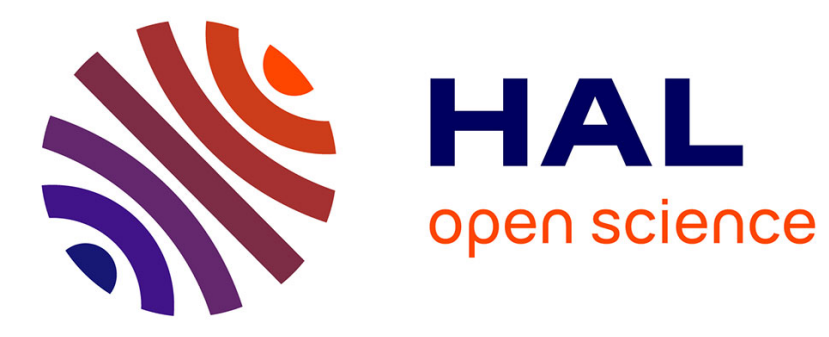

\title{
Improving the Efficiency of Traditional DTW Accelerators
}

\author{
Romain Tavenard, Laurent Amsaleg
}

\section{To cite this version:}

Romain Tavenard, Laurent Amsaleg. Improving the Efficiency of Traditional DTW Accelerators. Knowledge and Information Systems (KAIS), 2015, 42 (1), pp.215-243. 10.1007/s10115-013-0698-7 . hal-00862176

\section{HAL Id: hal-00862176 https://hal.inria.fr/hal-00862176}

Submitted on 22 Feb 2017

HAL is a multi-disciplinary open access archive for the deposit and dissemination of scientific research documents, whether they are published or not. The documents may come from teaching and research institutions in France or abroad, or from public or private research centers.
L'archive ouverte pluridisciplinaire HAL, est destinée au dépôt et à la diffusion de documents scientifiques de niveau recherche, publiés ou non, émanant des établissements d'enseignement et de recherche français ou étrangers, des laboratoires publics ou privés. 


\title{
Improving the Efficiency of Traditional DTW Accelerators
}

\author{
Romain Tavenard ${ }^{1}$ and Laurent Amsaleg ${ }^{2}$ \\ ${ }^{1}$ Idiap Research Institute* \\ Rue Marconi, 19 - 1920 Martigny, Switzerland \\ Romain.Tavenard@idiap.ch \\ Phone: +41 277217785 \\ Fax: +41 277217712 \\ 2IRISA / CNRS, France \\ Campus universitaire de Beaulieu - 35042 Rennes cedex, France \\ *This work has been conducted while Romain Tavenard was pursuing his Ph.D. at INRIA \\ Rennes with a scholarship from Université de Rennes 1.
}

\begin{abstract}
Dynamic time warping (DTW) is the most popular approach for evaluating the similarity of time series, but its computation is costly. Therefore, simple functions lower bounding DTW distances have been designed, accelerating searches by quickly pruning sequences that could not possibly be best matches. The tighter the bounds, the more they prune and the better the performance. Designing new functions that are even tighter is difficult because their computation is likely to become complex, canceling the benefits of their pruning. It is possible, however, to design simple functions with a higher pruning power by relaxing the no false dismissal assumption, resulting in approximate lower bound functions. This paper describes how very popular approaches accelerating DTW such as LB_Keogh and LB_PAA can be made more efficient via approximations. The accuracy of approximations can be tuned, ranging from no false dismissal to potential losses when aggressively set for great response time savings. At very large scale, indexing time series is mandatory. This paper also describes how approximate lower bound functions can be used with $i$ SAX. Furthermore, it shows that a $k$-means-based quantization step for $i \mathrm{SAX}$ gives significant performance gains.
\end{abstract}

Keywords: Dynamic time warping; Indexing; Lower bounds; Upper bounds; Indexing Trees

Received Feb 22, 2012

Revised Apr 26, 2013

Accepted Sep 14, 2013 


\section{Introduction}

Searching in very large databases of time series has received a lot of attention from researchers. Dynamic time warping (DTW) has proved to be an excellent similarity measure for time series. It has been successfully applied to domains as diverse as pattern recognition (Munich and Perona, 1999), life sciences and bioinformatics (Aach and Church, 2001; Gavrila and Davis, 1995), speech recognition (Itakura, 1975), etc. DTW is of great help when using any data represented as a sequence for discovering motifs or rules, for clustering or classifying sequences, or for answering query by contents.

DTW finds the optimal alignment between two given time series. One of its strength is to cope with local distortions along the time dimension. DTW, however, is slow and costly to calculate as its time complexity is quadratic. Therefore, three families of approaches have been defined to accelerate DTWbased searches.

\subsection{Lower-Bounding Functions}

The first family of approaches includes cheap-to-compute functions lower bounding DTW (Keogh and Ratanamahatana, 2005; Yi, Jagadish and Faloutsos, 1998). They accelerate the DTW process because they quickly prune sequences that could not possibly be a best match. Then, the full DTW is computed on the list of candidate sequences that remain. Lower bound functions have two key properties: (i) They incur no false dismissals as suggested in Faloutsos, Ranganathan and Manolopoulos (1994) and (ii) the tighter they are, the more they prune. At a very fine level, even LB_Keogh, known to be the tightest lower bound in the literature, can be quite far from the true value of the DTW (Keogh and Ratanamahatana, 2005). There is therefore room for improvement, as a tighter bound would allow more pruning and faster searches. Yet, designing a tighter lower bound function is difficult as its computation is likely to become complex, and thus less profitable.

The first contribution of this paper is the definition of approximate lower bounding functions built on top of the popular LB_Keogh and LB_PAA (exact) lower bounds. These approximate lower bounding functions have a user-tunable tightness: It can range from the one achieved by their exact counterpart, and in this case, no false dismissals are observed, to much tighter bounds where false dismissals are possible as the DTW may get over estimated.

\subsection{Indexing Time-Series}

The second family of approaches includes schemes specifically designed to cope with very large databases of time series. Camerra, Palpanas, Shieh and Keogh (2010), Shieh and Keogh (2008), Keogh and Ratanamahatana (2005) and Vlachos, Hadjieleftheriou, Gunopulos and Keogh (2003) all index time series for improved performance. Indexing shrinks the search space to some neighborhood defined around the query sequence, which, in turn, dramatically reduces the response time. Not surprisingly, lower bounding functions are at the core of such indexing schemes for even better performance. For example, Lin, Keogh, Wei 
and Lonardi (2007) define $i$ SAX_MinDist that prunes sequences based on the range intervals determined for quantizing time series.

The second contribution of this paper is the definition of an approximate lower bounding function used in $i \mathrm{SAX}$. It builds on $i \mathrm{SAX}$ _MinDist, and its tightness can also be tuned by the users to offer a wide range of response time versus accuracy trade-offs.

The performance of $i \mathrm{SAX}$ depends on the one hand on the lower bounding function used, and on the other, it depends on the way the tree used for indexing is built. $i \mathrm{SAX}$ and $i \mathrm{SAX} 2.0$ quantize time series in a tree of symbols. As discussed in Camerra et al. (2010), the quantization intervals are determined in order to be as balanced as possible assuming the data in time series are normally distributed. This is, in fact, rarely the case.

The third contribution of this paper is the use of a $k$-means-based quantization step for $i \mathrm{SAX}$. Using $k$-means avoids to make any strong assumption on the distribution of data in time series. The resulting index better fits data. Some special care is taken to balance the tree of symbols, as this is key to performance.

\subsection{Approximate Searches}

The third family has approaches that have traded accuracy for response time (Chu, Keogh, Hart, Pazzani et al., 2002; Shieh and Keogh, 2008). In this case, great performance gains are obtained at the cost of some potential false dismissals. What is central to these schemes is the metrics they use for assessing the similarity of time series, metrics that are no longer lower bounding the DTW. In addition to its exact $i$ SAX_MinDist, $i$ SAX defines such an approximate metrics. Shieh and Keogh (2008) show the approximate version of $i$ SAX can answer a query in less than a second, while exact $i \mathrm{SAX}$ requires about $10 \mathrm{~min}$.

The last contribution of this paper is to show how approximate lower bounds can be used to boost the performance of the approximate version of $i \mathrm{SAX}$.

\subsection{Discussion}

Experiments made with state-of-the-art datasets (Keogh, Xi, Wei and Ratanamahatana, 2006) show that these contributions significantly accelerate DTW. When considering small datasets, sequentially scanning the data collection is still appropriate. In this case, using approximate lower bounding functions is an order of magnitude faster than using exact lower bounds while indeed very rarely triggering false dismissals. It is twice as fast as Iterative deepening dynamic time warping (IDDTW) (Chu et al., 2002) while achieving comparable recall performance.

Performance gains are even more significant when considering large datasets for which indexing is needed. Approximating $i$ SAX_MinDist allows to run $i$ SAX two order of magnitude faster than its exact version while still returning one of the 50 actual nearest neighbors at rank 1 in $87 \%$ of the cases. Performance gains are about one order of magnitude compared to the approximate version of $i \mathrm{SAX}$ while achieving comparable retrieval performance, quality-wise. These approximate methods can deal with applications for which exact searches would be far too long to perform.

This paper is structured as follows. Section 2 gives the necessary background 
on the DTW and on few popular techniques boosting its performance. Section 3 details how these techniques can be improved thanks to approximate lower bounding functions. Section 4 introduces $i \mathrm{SAX}^{+}$, an indexing tree borrowing from $i \mathrm{SAX}$ that, however, relies on $k$-means clustering for determining quantization intervals. Note these two sections give pseudocodes. Section 5 presents the extensive experiments made using the datasets of Keogh and Ratanamahatana (2005) and (Shieh and Keogh, 2008), showing the algorithms we propose here outperform state-of-the art solutions. Section 6 concludes.

\section{Accelerating DTW}

This section reviews the background material needed for this paper. We first give a quick description of the DTW process. We then describe four very classical methods that have been designed to accelerate its computation. We then discuss IDDTW (Chu et al., 2002) which approximates DTW searches.

\subsection{DTW}

It has been demonstrated that similarity searches in time series is best when using DTW. DTW takes two time series, $Q$ and $C$, of length $m$ and $l$ respectively, where

$$
Q=q_{1} q_{2} \ldots q_{m} ; C=c_{1} c_{2} \ldots c_{l}
$$

To evaluate the similarity between $Q$ and $C$, a $m \times l$ matrix $S$ is computed such that:

$$
\forall(i, j) \in\{1, \ldots, m\} \times\{1, \ldots, l\}, S_{i, j}=d\left(q_{i}, c_{j}\right)
$$

where $d\left(q_{i}, c_{j}\right)$ is the distance between $q_{i}$ and $c_{j}$. A warping path $W$ is the set of $K$ elements of $S$ forming a path $W=w_{1} \ldots w_{K}$ where $w_{k}=(i, j) \in$ $\{1, \ldots, m\} \times\{1, \ldots, l\} . W$ has to meet three conditions: It has to be continuous, monotonic and has to meet boundary conditions (it goes from $\left(q_{1}, c_{1}\right)$ to $\left(q_{m}, c_{l}\right)$ ).

Dynamic programming techniques are used to efficiently find this path via a recurrence over a cumulative distance $\gamma_{i, j}$ defined as follows:

$$
\forall(i, j) \in\{1, \ldots, m\} \times\{1, \ldots, l\}, \gamma_{i, j}=\min \left\{\begin{array}{l}
S_{i, j}+\gamma_{i-1, j-1} \\
S_{i, j}+\gamma_{i-1, j} \\
S_{i, j}+\gamma_{i, j-1}
\end{array}\right.
$$

A direct implementation of DTW has a time and space complexity of $O(m \times l)$.

It has been shown that for some applications, restricting the admissible paths around the diagonal could be beneficial in terms of both complexity and relevance of the resulting metrics. The most commonly used constraints are the SakoeChiba band (Sakoe and Chiba, 1978) and the Itakura parallelogram (Itakura, 1975). The former constrains the admissible paths to lay inside a band of fixed width around the diagonal path, as shown in Fig. 1, while the latter uses a parallelogram that has the diagonal path as one of its diagonals. 


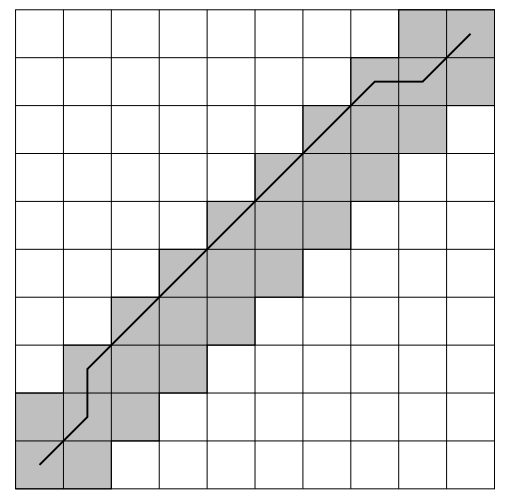

(a) Sakoe-Chiba band

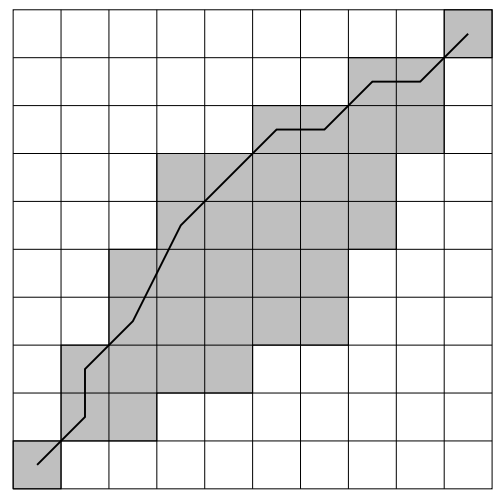

(b) Itakura parallelogram

Fig. 1. Examples of constrained DTW paths. Admissible paths are restricted to the gray areas.

\subsection{Accelerator \#1: LB_Keogh}

LB_Keogh (Keogh and Ratanamahatana, 2005) is probably the best-known lower bound function. LB_Keogh $(Q, C)$ first rescales $Q$ and $C$ to the same length $n$, then builds the bounding envelope of $Q$ by determining all its maximum and minimum values inside a Sakoe-Chiba band (Sakoe and Chiba, 1978) (or an Itakura parallelogram (Itakura, 1975)). It then sums the distances from every part of $C$ not falling within the bounding envelope of $Q$ to the nearest point from that envelope having the exact same time stamp. Keogh and Ratanamahatana (2005) show, for any $Q$ and $C$ rescaled that LB_Keogh $\leq$ DTW. The complexity of LB_Keogh is linear in $n$.

\subsection{Accelerator \#2: LB_PAA}

LB_PAA is another approach further reducing the cost of computing LB_Keogh. LB_PAA first requires to chop all sequences $C$ stored in the database into $N$ chunks, containing a number of points that depends on the length of considered sequences. This creates sequences downsampled along their timeline. Downsampled versions of $C$ are then compared to a downsampled version of the envelope of $Q$ in a manner that is similar to the one for LB_Keogh. This gives the LB_PAA function, lower bounding LB_Keogh, and, therefore, DTW. Note the complexity of LB_PAA is equivalent to the complexity of computing LB_Keogh for time series of length $N$, which is smaller than $n$ and thus computing LB_PAA is more efficient than computing LB_Keogh.

\subsection{Accelerator \#3: $i \mathrm{SAX}$}

Both LB_Keogh and LB_PAA must be computed for each sequence $C$ from the database to find the one that is the most similar to $Q$. Researchers have thus worked on finding indexing techniques where only a small subset of relevant sequences from the database would have to be compared to $Q$, which would in turn prune the search space even more. 
Since LB_PAA turns sequences into fixed-length descriptions lying in a $N$ dimensional space, it is possible to index them, typically in a R-Tree, as proposed in Keogh and Ratanamahatana (2005). The index eventually contains minimum bounding rectangles (MBR) in which similar sequences are grouped. At query time, the algorithm finds the sequences from the database that are close to $Q$ using the proximity of their respective MBR, quickly determined through the index.

Building on this, an even more efficient DTW indexing scheme called iSAX was proposed in Shieh and Keogh (2008). iSAX also downsamples sequences, but it additionally quantizes them. $i$ SAX thus gives a discrete representation to continuous sequences. It turns time sequences into a series of symbols that can easily be indexed in a tree. The quantization boundaries giving symbols for the values are determined according to a standard normal distribution. The size of the alphabet is dynamically adjusted during index construction to balance symbols. iSAX was later extended into iSAX2.0 (Camerra et al., 2010), that achieves more efficient index construction thanks to bulk loading and more balanced clusters via a smart symbol selection scheme to use when splitting a node from the tree.

In this case, the $i$ SAX_MinDist metrics defined in Shieh and Keogh (2008) that is used to assess similarity between a query and a node of the tree is in the same spirit as the ones presented above, except that the ranges assigned to nodes are used to replace MBRs. The search process then consists of visiting each leaf of the tree in ascending order of their $i$ SAX_MinDist values, and the algorithm stops as soon as all remaining leaves in the tree have $i$ SAX_MinDist values greater than the current best distance found.

\subsection{Accelerator \#4: Approximate $i \mathrm{SAX}$}

Shieh and Keogh (2008) also present a slight variant of the exact $i$ SAX version that uses $i$ SAX_MinDist. This variant returns approximate results as it does not scan all theoretically possible leaves, but solely the one leaf with the lowest lower bound. That single leaf is entirely scanned, and LB_Keogh is determined for each sequence, which possibly prunes some sequences from any further analysis. As usual, full DTW are computed for the remaining sequences from that leaf to build the final result returned to the user. Returning data from that single leaf is of course very fast. Searching the index for time series that are similar to the query thus aborts as soon as the most probable leaf from the tree has been scanned. For the sake of clarity, we call this way of accelerating the searches iSAX_Approx.

\subsection{IDDTW}

Iterative deepening dynamic time warping (IDDTW) (Chu et al., 2002) aims, as previously introduced lower bounds for DTW, at computing DTW only for those candidate sequences in the database that are likely to be similar to the considered query. This likelihood is estimated by computing DTW between downsampled versions of the sequences. The principle is then to start comparing sequences at a very coarse resolution and keep refining the latter until the sequence can be discarded from the candidate list or, if not, until full resolution is reached. To 
test if a sequence can be discarded at a given resolution, a learned distribution of the estimation error at this resolution is used. Given this error distribution, if the probability that the computed estimation can lead to a lower DTW value than that of the best sequence so far is lower than a user-defined threshold, the sequence is discarded. If not, this process is repeated at a finer resolution.

This algorithm heavily relies on probabilistic estimation. Therefore, the false dismissal assumption is discarded for the sake of lower computational cost.

\section{Improving accelerators via approximations}

This section describes how the efficiency of the four DTW accelerators can be improved thanks to approximations. It first describes how the $\mathcal{A} \_$Keogh and the $\mathcal{A}$ _PAA approximate lower bound functions can be defined ${ }^{1}$ from their exact counterparts LB_Keogh and LB_PAA. This improves the performance of the accelerators $\# 1$ and $\# 2$. Then, this section moves to defining $\mathcal{A} \_i$ SAX which improves the performance of the accelerator \#3, indexing DTW via $i$ SAX. It also defines $\mathcal{A} \_i$ SAX_Approx, built from $i$ SAX_Approx which enhances the performance of Approximate $i$ SAX. This section ends by presenting the pseudo-codes for two algorithms derived from that of Keogh and Ratanamahatana (2005) and Shieh and Keogh (2008), one for a sequential-scan-based search algorithm, the other for an index-based search.

\section{1. $\mathcal{A} \_$Keogh}

The pruning power of LB_Keogh is directly linked to its tightness, that is the ratio of lower bound value to actual DTW value. The tighter it is, i.e., the closer to the real value of the DTW the lower bounding values are, the better. Given a time series, computing its LB_Keogh value does not help in knowing whether it is tight, i.e., close or far from its actual DTW value. It is not the value of LB_Keogh that matters, but the relative difference between that value and the true value of DTW. It has been observed that LB_Keogh is often quite smaller than the true value of the DTW, especially with rapidly varying sequences. What is key is to have a way to distinguish the case where (i) the value of the lower bounding function is small because the DTW is also small, from the case where (ii) it is small because its calculation is poorly tight.

To reach that goal, we first explain why applying a single multiplicative factor to the obtained lower bound is not enough. Given that, we describe the design of a cheap-to-compute upper bounding function that is very similar in spirit to LB_Keogh. This upper bound is used to get an indication on the tightness of the lower bounding function LB_Keogh. By repeatedly computing the lower and upper bounds on a first set of sequences (denoted in the following as the learning set), it is possible to learn and then model the overall tightness. We then introduce a mechanism to determine, given a tunable parameter that is closely linked to the tightness model, how many DTW values are likely to be overestimated. Overall, this yields approximate lower bounding functions. Thanks to the model for overestimations, it is possible to fine tune the pruning power of

\footnotetext{
$\overline{1}$ The $\mathcal{A}_{\text {- prefix stand for approximate. }}$
} 


\begin{tabular}{cccc}
\hline Function & $\begin{array}{c}\text { Median } \\
\text { tightness } \\
\text { (learning) }\end{array}$ & $\begin{array}{c}\text { Median } \\
\text { tightness } \\
\text { (test) }\end{array}$ & $\begin{array}{c}\text { Quartiles } \\
\text { of tightness } \\
\text { (test) }\end{array}$ \\
\hline LB_Keogh & 0.857 & 0.916 & {$[0.500 ; 0.994]$} \\
$\alpha$ LB_Keogh & $(0.99)$ & 1.058 & {$[0.578 ; 1.148]$} \\
$\mathcal{A} \_K e o g h$ & $(0.99)$ & 0.993 & {$[0.929 ; 1.000]$} \\
\hline
\end{tabular}

Table 1. Observed median of $T$ values and its quartiles when the input parameter is set so as to imitate a magic lower bound $99 \%$ tight. Note that for $\alpha$ LB_Keogh and $\mathcal{A} \_$Keogh, learning values are given between parenthesis as their parameters are set so as to get $99 \%$ median tightness on the learning set.

such functions: very aggressive functions that are likely to overestimate DTW will have a very good pruning power, but may in turn create many false dismissals, in contrast to milder functions.

\subsection{1. $\alpha L B \_K e o g h$}

As explained above, it has been observed that LB_Keogh is often quite smaller than the true value of the DTW, especially when dealing with rapidly varying sequences. A first option to approximate existing lower bounds would have been to learn the typical ratio between DTW values and lower bound ones using a large set of diverse sequences and a set of representative queries. It would then be sufficient to compute a multiplication factor $\alpha$ to apply to LB_Keogh during the search process in order to increase the tightness of a new approximate lower bounding function that we call $\alpha$ LB_Keogh.

We show that this idea leads to poor approximations through experimentation. Since we believe that at large scale, a database of sequences is highly diverse, we merged into a single database all the datasets listed in Keogh and Ratanamahatana (2005) (that will later be referred to as dataset $\mathrm{DS}_{1}$ ) and set parameter $\alpha$ in order to achieve $99 \%$ tightness on the learning set.

The first two rows of Table 1 show that the median value for tightness is around $92 \%$ for LB_Keogh, meaning that the returned value is, in median, $8 \%$ lower than that of the DTW. Note also that $\alpha$ LB_Keogh tends to overestimate DTW, its median tightness being around 105\%. Moreover, interquartiles intervals are large for these two methods, which implies, for LB_Keogh, a high number of sequences for which the DTW is underestimated and, for $\alpha$ LB_Keogh, a high number of sequences for which the DTW is overestimated. This shows that a single multiplicative factor is not sufficient to build a reliable approximative lower bound. Indeed, using $\alpha$ LB_Keogh would only slightly increase tightness when the ratio between DTW and LB_Keogh is large, while it will return a value that would be too high when the ratio tends towards 1.

\subsubsection{UB_Keogh: an Envelope-based Upper Bound}

Vlachos et al. (2003) and Kashyap and Karras (2011) propose to use an upper bound to prune sequences that could not match a given query. In this paper, we are using an upper bound not for pruning, but to determine the interval (between the lower and upper bounds) within which the true value of the DTW is. If the interval is small, then it is likely the lower and upper bounds are tight.

One straightforward way to upper bound the DTW is to compute the Man- 


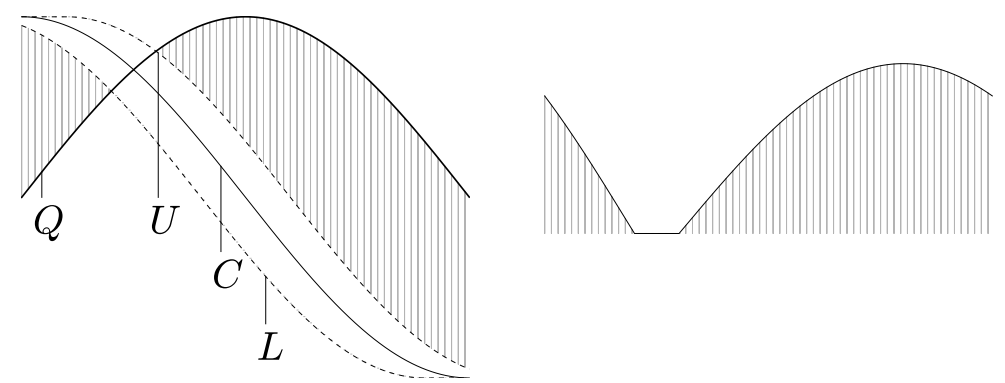

(a) LB_Keogh $(Q, C)$
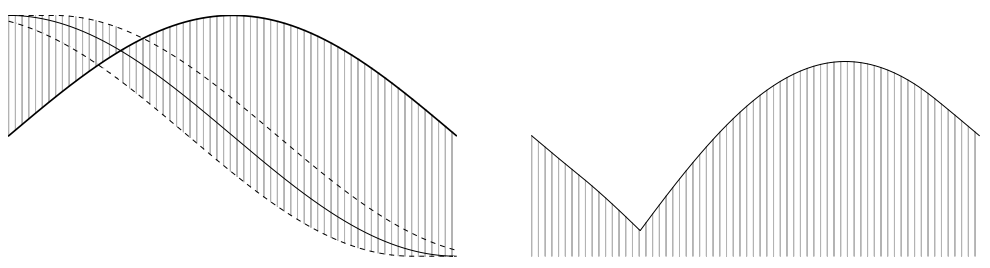

(b) UB_Keogh $(Q, C)$

Fig. 2. Example of LB_Keogh and UB_Keogh. $U$ and $L$ are defined as in Keogh and Ratanamahatana (2005). The right hand side of each figure shows the overall area accounted for when computing each bound.

hattan distance ${ }^{2}$ between $Q$ and $C$. A more elegant way is to rely on the envelopes around sequences that are already used for computing lower bounds. We experimentally observed that the intervals around the true values of the DTW are more stable when the bounds are defined according to the same underlying principle (envelopes). In addition, as it will be detailed later in this paper, relying on envelopes facilitates the computation of an upper bound on downsampled data as it is the case for LB_PAA.

Building on LB_Keogh, it is possible to define an envelope-based upper bound, called UB_Keogh. UB_Keogh is computed by summing the distances between every point in the query and the furthest point having the exact same time stamp on the envelope of each database sequence. The mathematical definition of UB_Keogh as well as the proof it truly upper bounds DTW is provided in the Appendix. By definition:

LB_Keogh $\leq$ DTW $\leq$ UB_Keogh.

The complexity of computing UB_Keogh is the same as the one of computing LB_Keogh, i.e., it is linear in $n$. Figure 2 gives a graphical illustration of LB_Keogh and UB_Keogh.

\footnotetext{
2 Note that, in this paper, we chose to rely on the DTW formulation that is used in the work of Sakoe and Chiba (1978), which leads to the Manhattan distance being an upper bound while, when using the same formulation as in Keogh and Ratanamahatana (2005), DTW is upper-bounded by Euclidean distance.
} 


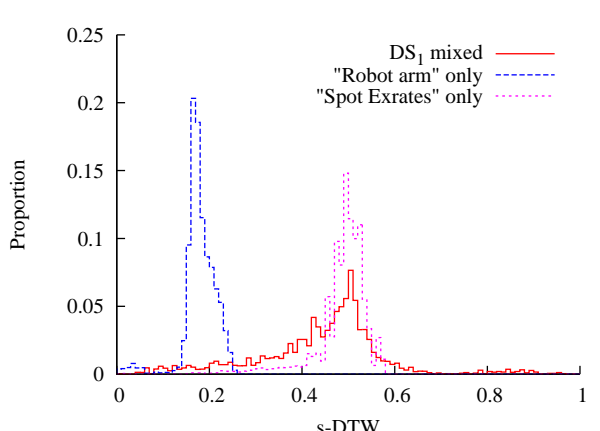

(a)

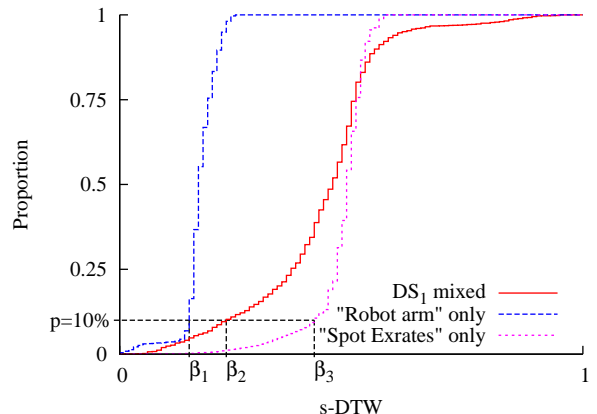

(b)

Fig. 3. Experimental probability density function (a) and cumulative density function (b) for the whole merged dataset $\mathrm{DS}_{1}$ and for its sub-datasets "Robot Arm" (labeled \#20 later) and "Spot Exrates" (labeled \#24 later).

When processing $Q$ and $C$, the difference between LB_Keogh and UB_Keogh gives a clear indication on the tightness of these bounds, a small difference suggesting that the bounds are tight.

\subsubsection{Modeling Tightness}

It is possible to learn what the values for LB_Keogh and UB_Keogh are, as well as the ones for the true DTW for each pair of sequences in the learning set. A histogram of the distribution of these values, once normalized, can then be computed. Normalized values are defined as follows:

$$
\mathrm{s}-\mathrm{DTW}(\mathrm{Q}, \mathrm{C})=\frac{\mathrm{DTW}(Q, C)-\mathrm{LB} \_\operatorname{Keogh}(\mathrm{Q}, \mathrm{C})}{\mathrm{UB} \_\operatorname{Keogh}(\mathrm{Q}, \mathrm{C})-\mathrm{LB} \operatorname{Keogh}(\mathrm{Q}, \mathrm{C})} \text {. }
$$

s-DTW is closely related to the tightness observed on the learning set: A left-shifted curve for the probability density function of s-DTW suggests most LB_Keogh values are tight, while most are loose when right-shifted. We validate this idea through experimentation. Since we believe that at large scale, a database of sequences is highly diverse, we merged into a single database all the datasets listed in Keogh and Ratanamahatana (2005) (that will later be referred to as dataset $\left.\mathrm{DS}_{1}\right)$.

Figure 3a shows, for $\mathrm{DS}_{1}$, the distribution of s-DTW over all sequences. It also plots distributions observed for two sub-datasets. Few comments are in order. First, all plots are away from the $x$-axis zero value, meaning that for every pair of sequences considered, LB_Keogh is strictly lower bounding DTW. This is basically the proof that there is room from improvement: it is possible to use a greater value than LB_Keogh (i.e. shifting decision value to the right) while still experiencing very few (or even no) DTW overestimations (shown on the $y$-axis of Fig. 3b). Second, there is a significant shift between the curves, which motivates the use of a learnt histogram modeling the s-DTWdistribution rather than a single shift value.

The distribution of s-DTW values is an indicator of the tightness observed on the learning set: We next show how it can be used as a model, to predict the tightness of sequences outside the learning set. 


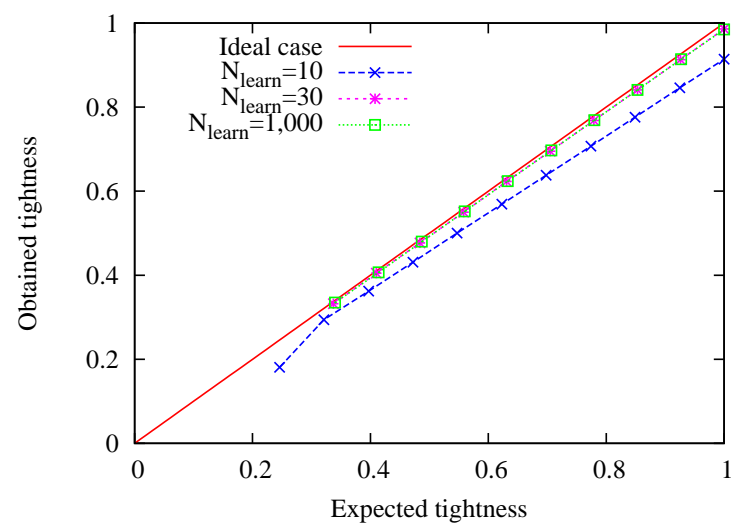

Fig. 4. Setting tightness as an input parameter. Here, a given tightness value is targeted using dichotomy and we study the behavior of $\mathcal{A} \_$Keogh with respect to the number of learning sequences. The dataset used for this experiment is the one called "Two_Patterns" from the larger dataset that will later be denoted as $\mathrm{DS}_{2}$.

\subsubsection{Determining the Over-Estimation Rate}

By computing the cumulative distribution of s-DTW, one can estimate the expected distance over-estimation rate. Assume one wants to speed up the search process at the price of potential false dismissals. That user decides to allow for $p=10 \%$ of over-estimated distances. Given the learned cumulative distribution, one can find the value of $\beta$ on the $\mathrm{x}$-axis that gives $p$ on the $\mathrm{y}$-axis (see the dashed lines on Fig. 3b). $\beta$ is then used to compute the approximate lower bound equal to

$$
\mathcal{A} \_ \text {Keogh }=\text { LB_Keogh }+\beta(\text { UB_Keogh }- \text { LB_Keogh })
$$

that will subsequently be used to prune sequences. $p$ might be meaningless for some users. Instead, they might prefer achieving a target tightness $T$. Finding the optimal value for $p$ in order to achieve a target tightness $T$ can be performed by using dichotomy, as explained in more details in Sect. 3.1.5. The last row of Table 1 provides indications on the observed tightness of $\mathcal{A} \_$Keogh when setting $p$ in order to target $99 \%$ tightness. The observed median tightness is very close to this target, and its interquartile interval is small, suggesting a robust estimation that backs the use of information from upper bounding functions to get a better estimation of the actual DTW value.

\subsubsection{Discussion}

In this section, we will discuss some of the salient properties $\mathcal{A} \_$Keogh has, making it special with respect to other lower bounding functions.

Learning from data To achieve better tightness, $\mathcal{A} \_$Keogh relies on the distribution of s-DTW values in the $[0 ; 1]$ interval. An estimate of this distribution is obtained by computing a histogram of such values on a learning set. Note that we do not aim at gathering general knowledge from the data, but rather dataset-specific information. Hence, one can either use a learning set if there is one available or build one by using a subpart of the dataset to be searched. 
Cost of off-line learning Building such a histogram requires off-line DTW computations. More precisely, a learning set made of $N_{\text {learn }}$ sequences implies $\frac{N_{\text {learn }}\left(N_{\text {learn }}-1\right)}{2}$ DTW computations that have to be done once and for all at the learning step. This fixed off-line cost is affordable and helps saving time for the critical phase during which user queries have to be processed in a timely fashion. Experimental results presented in this paper do not refer to this cost as we believe the on-line query cost is the key for such methods. To illustrate that few learning sequences are required for our method to work properly, we show in Fig. 4 the difference between expected and obtained tightness. In this case, a learning set made of 30 sequences is sufficient to achieve a good modeling of tightness since the bias that can be observed in this case is still present when using 1,000 learning sequences, which means it is due to the bias between the learning and test sets. In this case, then, $\frac{30 \times 29}{2}=435$ off-line DTW computations are sufficient to learn characteristics of a dataset that is made of 1,000 learning sequences.

Moreover, the number of sequences used for learning drives the resolution of parameter $p$, as the latter is determined by the resolution of the cumulative density function learned on these sequences. A reasonable condition is that the given $p$ value corresponds to at least one observation in the training set:

$$
p \cdot \frac{N_{\text {learn }}\left(N_{\text {learn }}-1\right)}{2} \geq 1,
$$

which is satisfied for

$$
N_{\text {learn }} \geq \frac{1}{2}+\sqrt{\frac{1}{4}+\frac{2}{p}}
$$

Using a typical value of $p=0.01$, this condition becomes $N_{\text {learn }} \geq 15$, which is satisfied by the learning set used in this section.

Setting an optimal value for $p$ Setting an optimal value for parameter $p$ depends on the kind of problem the user wants to tackle: When dealing with relatively small datasets, she might want to achieve very good accuracy results, as the query cost is not a strong issue in this case, whereas when dealing with very large datasets, setting a large value for $p$ might be the only way to get a result in an acceptable amount of time. Finally, note that, when setting $p=0$, $\mathcal{A} \_$Keogh $=$LB_Keogh.

Setting a constraint on $T$ As stated above, it might be more meaningful for a user to set an expected value for $T$ rather than for $p$. This can be done by using dichotomy as the function of $T$ giving $p$ is nondecreasing. Indeed, tightness is defined as the ratio of $\mathcal{A} \_$Keogh to DTW, and is then a nondecreasing function of $\beta$ ( $c f$ Equation 6). As $\beta$ is a nondecreasing function of $p$ (this comes from the definition of a percentile), we get that tightness is a nondecreasing function of $p$. Dichotomy consists in recursively splitting the $[0,1] p$ value interval into smaller ones. To do so, at each step, the current interval is divided into two parts, and the middle of the interval is used as a $p$ value. If the tightness computed on the learning set for this $p$ value is lower than the targeted one, then the upper half interval is kept for the next iteration; otherwise, the lower half interval is considered. This process is performed on the learning set so as to learn a lookup table that can be used afterward for the user to give $T$ as an input. 


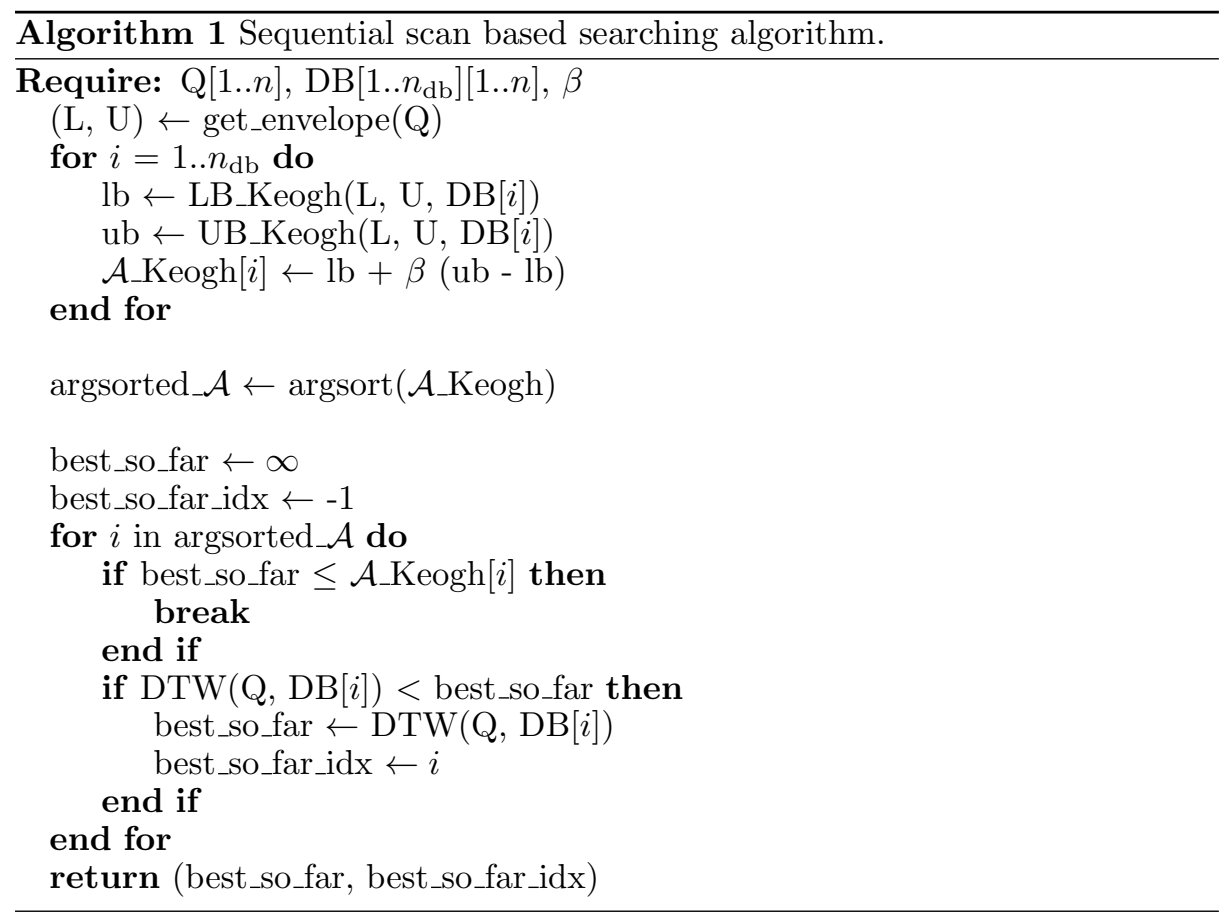

The effectiveness of this process is illustrated in Fig. 4, which shows that this dichotomy process is efficient even if using very few learning sequences.

If the user prefers to set a complexity constraint in terms of the expected amount of DTW computations, it is then possible to set the value for $p$ by numerically inverting the relationship between this amount of computations and $p$. The proof that the amount of DTW computations is a nondecreasing function of $p$ comes from the fact that this amount is a nondecreasing function of the tightness that has been shown earlier in this section to be a nondecreasing function of $p$.

\section{2. $\mathcal{A} \_$PAA}

The previous section defined UB_Keogh and explained how to calculate $\mathcal{A} \_$Keogh. It is possible to obtain UB_PAA and $\mathcal{A} \_$PAA in a very similar manner. Few comments are in order, however. First, LB_PAA downsamples all sequences into $N$ chunks. The envelope of $Q$ is therefore defined according to its downsampled version. UB_PAA is thus also defined on this downsampled version. Mathematical definition of UB_PAA is in the Appendix. Second, LB_PAA $\leq$ DTW $\leq$ UB_PAA. Last, the formula for modeling tightness (see Eq. (5)) has to be slightly changed in a trivial manner to use LB_PAA and UB_PAA. Note also that when the rate of DTW over estimations $p$ is set to 0 , then $\mathcal{A}_{-}$PAA $=$LB_PAA. 


\section{3. $\mathcal{A} \_K e o g h$ and $\mathcal{A} \_P A A$ for Sequential Scan}

LB_Keogh and LB_PAA are defined in Keogh and Ratanamahatana (2005). They are both used together with a search algorithm doing a sequential scan of the entire collection. Either lower bounds are computed on every sequence $C$ from the database to quickly get it compared to $Q$.

This section presents a slight variant of this sequential scan search process that uses either LB_Keogh, UB_Keogh and $\mathcal{A} \_K e o g h$ or LB_PAA, UB_PAA and $\mathcal{A} \_$PAA. The pseudo-code in this algorithm focuses on $\mathcal{A} \_$Keogh. It is straightforward to adapt to $\mathcal{A} \_$PAA.

Prerequisites $n_{\mathrm{db}}$ sequences are stored in a database DB. Each sequence has been rescaled to length $n$.

Off-Line Learning For each pair of sequences $\left(C_{i}, C_{j}\right)$ from the learning set, DTW, LB_Keogh, UB_Keogh and s-DTW are computed. Then, the average cumulative distribution for s-DTW is calculated, and a lookup table $\mathcal{C}$ keeping track of the quantiles of this distribution is built.

Query Time User Input The user inputs are $Q$ and $p$. $Q$ is the query, and it gets rescaled to length $n . p$ is the average overestimation rate. $p$ is used to probe $\mathcal{C}$, resulting in the target quantile $\beta$.

On-Line Searching The sequential-scan-based searching algorithm is detailed in Algorithm 1. An important point here is that when setting $\beta>0$, the break instruction can be triggered before finding the exact nearest neighbor.

\section{4. $\mathcal{A} \_i \mathrm{SAX}$ and $i \mathrm{SAX}$ indexing}

Indexing sequences is needed at large scale. The most popular time series indexing scheme is probably $i$ SAX (Shieh and Keogh, 2008). For efficiency, iSAX uses a lower bound function called $i$ SAX_MinDist. Following the principles defined above, it is possible to create an approximate lower bound function $\mathcal{A}_{-} i \mathrm{SAX}$ built on top of $i$ SAX_MinDist.

The definition of $i$ SAX_MinDist is slightly more complex compared to LB_Keogh or LB_PAA as it relies on the intervals defined during the index tree construction by the quantization process. With iSAX it is possible that some intervals associated with nodes in the index tree have infinite bounds. In this case, computing an upper bound is problematic. To facilitate this computation, we had to slightly modify the tree building process by storing, for each node that has at least one infinite bound, the corresponding extremum value for the sequences that are stored in the node or one of its children nodes. Therefore, the upper bound will use the actual extremum value instead of the infinite bound in its formula. That upper bound is called $i$ SAX_MaxDist and its mathematical definition is in the Appendix. Then, $i$ SAX_MinDist and $i$ SAX_MaxDist are used seamlessly in Eq. (5), eventually defining $\mathcal{A} \_i$ SAX.

The resulting $i \mathrm{SAX}$ index-based searching algorithm is detailed in Algorithm 2. Note this algorithm uses $i$ SAX_MinDist, $i$ SAX_MaxDist and $\mathcal{A}_{\_} i \mathrm{SAX}$ as well as the sequential scan algorithm presented above (and thus is relies on A_Keogh). 
Prerequisites $n_{\mathrm{db}}$ sequences are stored in a database DB. Each has been downsampled to length $N$.

Off-Line Indexing iSAX 2.0 off-line indexes all sequences as in Camerra et al. (2010).

Off-Line Learning Same as in Sect. 3.3. Additionally, however, quantiles for $\mathcal{A} \_$SAX and $\mathcal{A} \_$Keogh must be learned as both lower bounding functions are used at query time.

User Input The user inputs $Q$ and $p$. The two quantiles $\beta_{\mathrm{LB}_{-} K e o g h}$ and $\beta_{i \text { SAX_MinDist }}$ are obtained from $p$ and $\mathcal{C}$.

On-Line Searching The index based searching algorithm is detailed in Algorithm 2. It is built on $i \mathrm{SAX}$ (see Shieh and Keogh (2008)), with specifics due to the use of approximate lower bounds. Note that, isax_approximate_search, called to initialize the process, returns the leaf node having a SAX signature that matches the one of the query ${ }^{3}$ and seq_scan is Algorithm 1.

\section{5. iSAX_Approx indexing}

Shieh and Keogh (2008) describe a variant of $i$ SAX that returns approximate results. We have presented this variant in Sect. 2.5 and called it $i$ SAX_Approx. $i$ SAX_Approx determines the one best iSAX-leaf, computes LB_Keogh on all sequences from that leaf for pruning, and then computes full DTW on the remaining sequences. As it uses LB_Keogh, it is possible to patch this algorithm such that it uses $\mathcal{A} \_$Keogh instead.

It is therefore quite easy to turn $i \mathrm{SAX} \_$Approx into $\mathcal{A} \_i$ SAX_Approx.

$i$ SAX_Approx is already an approximate search scheme, due to its leaf picking strategy. $\mathcal{A} \_i$ SAX_Approx somehow piles up another layer of approximation as it uses $\mathcal{A} \_$Keogh. Using $\mathcal{A} \_$Keogh at the heart of $i$ SAX_Approx typically divides by 7 the number of sequences that go through a full DTW process for comparable recall performances. The pseudo-code for this $\mathcal{A} \_i$ SAX_Approx strategy is simply made of the four first lines of Algorithm 2 as it directly returns (idx_bsf, dist_bsf) once the best $i$ SAX node scanned by Algorithm 1 .

\section{4. $i \mathrm{SAX}^{+}$: Quantizing with $k$-means}

$i \mathrm{SAX}$ is a very successful approach because it turns continuous time series into discrete sequences of symbols, for which efficient high-dimensional indexing schemes exist. The cornerstone of $i \mathrm{SAX}$ is therefore its quantization process which is clearly defined in Camerra et al. (2010). It makes one strong assumption: The data in time series before quantization is assumed to be normally distributed. From that assumption, $i$ SAX determines the quantization intervals

3 There exists exactly one such node. isax_approximate_search is in fact the method defined in Shieh and Keogh (2008) and used in the next section describing $i$ SAX_Approx indexing. 


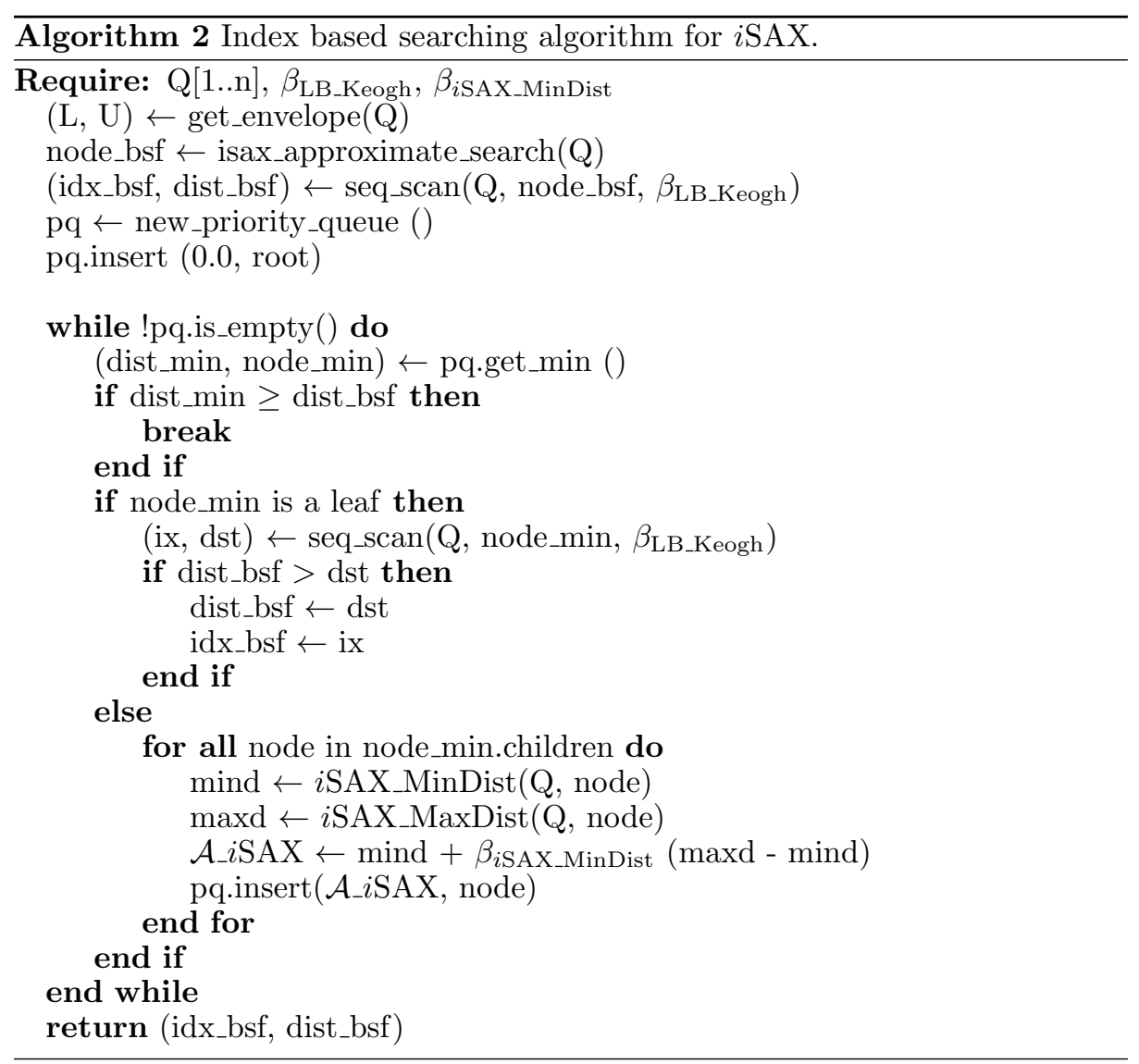

to balance the probability with which symbols will appear in the quantized sequences. Camerra et al. (2010) show it is key for performance to have (roughly) equiprobable symbols.

Unfortunately, the data in real-world time series are unlikely to follow a normal distribution. Note the biggest datasets used in Camerra et al. (2010) are synthetic and have been created so that considered time series have marginal normal distribution. As real-world time series rarely stick to a normal distribution, quantifying them as iSAX does is unlikely to give equiprobable symbols, which, in turn, hurts performance.

It is possible to relax this normal distribution assumption by using a variant of the $k$-means approach. $k$-means is a widely used unsupervised classification algorithm that takes as input a set of points $\mathbf{x}$ and a number $k$ of clusters to build. It makes no assumption on the distribution of data. It tends to minimize intra-cluster variance defined as follows:

$$
V=\sum_{i=1}^{k} \sum_{\mathbf{x} \in C_{i}}\left\|\mathbf{x}-\mathbf{c}_{\mathbf{i}}\right\|^{2}
$$

where $\mathbf{c}_{\mathbf{i}}$ is the centroid of cluster $C_{i}$.

Algorithms belonging to the $k$-means family have been used as unstructured 
quantizers in the context of image and video search (Sivic and Zisserman, 2003; Nistér and Stewénius, 2006; Paulevé, Jégou and Amsaleg, 2010; Tavenard, Jégou and Amsaleg, 2011). In this context, $k$-means has proved to nicely fit the data in space. This is the rationale for using $k$-means for quantizing time series. $k$-means, however, is also known to create Voronoi cells having rather uneven cardinalities. The variant we use here not only does $k$-means but also balances the clusters it creates. We now detail the way $k$-means can be used for $i \mathrm{SAX}$ as well as the cluster balancing strategy.

\section{1. $k$-means for Building the $i \mathrm{SAX}$-Tree}

The original $i \mathrm{SAX}$ tree forms a hierarchy of nodes. We therefore use $k$-means in a hierarchical way (Nistér and Stewénius, 2006) to get a similarly shaped index tree. The root node of the original $i \mathrm{SAX}$ index tree has $b^{w}$ children, where $b$ is the base cardinality of symbols and $w$ is the word length, i.e., the number of symbols in a sequence. Therefore, the root node when using the $k$-means approach has the same number of children nodes and results from determining $k=b^{w}$ centroids. Other levels below in the tree are made by running $k$-means with $k=2$ as for the original $i \mathrm{SAX}$ which has also 2 child nodes per parent node.

As it is the case for the original $i \mathrm{SAX}$ approach, building an index tree with $k$-means requires to split a node into two child nodes once the parent node gets fully filled with sequences. This very traditional recursive node splitting process eventually creates leaf nodes containing at most th sequences. Not surprisingly, a node keeps track of its MBR and its associated centroid, that centroid being produced by $k$-means. Inserting a new sequence in the index tree is performed by going down the tree and, at each level, by determining the centroid that is the closest to the sequence to insert. If that sequence has to be inserted in an entirely filled node, then a split occurs. $k$-means is applied to the sequences in that node that are then moved to child nodes according to the centroids newly determined.

\subsection{Balancing the $k$-means-based $i \mathrm{SAX}$-Tree}

It has been shown in Tavenard et al. (2011) that a $k$-means computed over a large set of high dimensional features produces clusters having quite different cardinalities. This, in turn, increases the response time variance of the algorithms processing the data in clusters, and performance might be particularly bad when using highly populated clusters. Having balanced clusters reduces this variance and improves performance, overall. Note this is also why the original version of $i$ SAX creates a balanced index tree.

Tavenard et al. (2011) balance the clusters produced by a $k$-means as follows. It first projects all the data points as well as the centroids computed by the $k$ means into a higher dimensional space. Then, it iteratively enlarges the distances between the centroid of a cluster and the corresponding data points in that cluster in proportion to the cardinality of the cluster. This moves inward the frontiers of highly populated clusters and reduces their cardinality because it assigns some of the points from that cluster to other, less crowded, clusters. Tavenard et al. (2011) can be run until all clusters contain the same number of points, or it can be stopped earlier, after a given number of iterations or when 
the standard deviation computed over the cardinalities of clusters falls below a threshold.

The mathematical formulas used in Tavenard et al. (2011) can be significantly simplified when the algorithm has to balance the cardinality of $k=2$ clusters. In this case, they can be turned into a simple algebraic equation given in the Appendix.

\section{3. $i \mathrm{SAX}^{+}$}

This section presents the pseudo-code for the complete $i \mathrm{SAX}^{+}$indexing scheme (Algorithm 3). It borrows a lot from iSAX. It makes use of $k$-means and of the procedure described in Tavenard et al. (2011) to create balanced clusters as described above.

The build_tree function is in charge of splitting overfilled nodes upon insertion. It uses the th parameter defined for the original $i \mathrm{SAX}$. At splitting time, the function split is invoked. It first quantizes the data using $k$-means. Then, it balances the children nodes using the mechanisms presented in Tavenard et al. (2011) and briefly sketched above.

Note the pseudo-code for querying the tree is not presented here as it is identical to Algorithm 2. The only (minor) difference lies in the approximate_search function that relies on the proximity of the centroids obtained by the $k$-means (and not on the set of symbols as for the original $i \mathrm{SAX}$ ).

\section{Experiments}

This section presents the extensive performance evaluation showing the techniques proposed in this paper outperform state-of-the art solutions. The first set of experiments compares LB_Keogh and $\mathcal{A} \_$Keogh, as LB_Keogh is the best exact lower bound ever proposed. This comparison involved evaluating their respective tightness and their resulting effectiveness in pruning more sequences. We also evaluate the impact of relaxing the no false dismissal assumption in the case of $\mathcal{A}_{-}$Keogh on the quality of the results returned to the user. Note this involves checking the ranking of candidate sequences that will go through a full DTW process as this ranking might differs from the one determined by LB_Keogh. Note LB_PAA and $\mathcal{A} \_P A A$ are also compared.

The second set of experiments compares $\mathcal{A} \_$Keogh with IDDTW. IDDTW also approximates DTW, but it uses downsampling instead of an approximate lower bound.

The third set of experiments focuses on the impact of the above techniques on $i \mathrm{SAX}$. It shows its performance is improved when using the approximate lower bounds $\mathcal{A}_{\_} i \mathrm{SAX}$ and $\mathcal{A} \_i \mathrm{SAX} \_$Approx.

Finally, we give the performance of $i \mathrm{SAX}^{+}$that relies on a balanced $k$-means quantization step.

For the sake of reproducibility of experiments, all evaluations are conducted using publicly available (Keogh et al., 2006) and widely used datasets (Keogh and Ratanamahatana, 2005; Shieh and Keogh, 2008). In all experiments, the baseline similarity measure is a DTW constrained by a Sakoe-Chiba band with a size set to $10 \%$ of the length of sequences, as suggested in Keogh and Ratanamahatana (2005). 


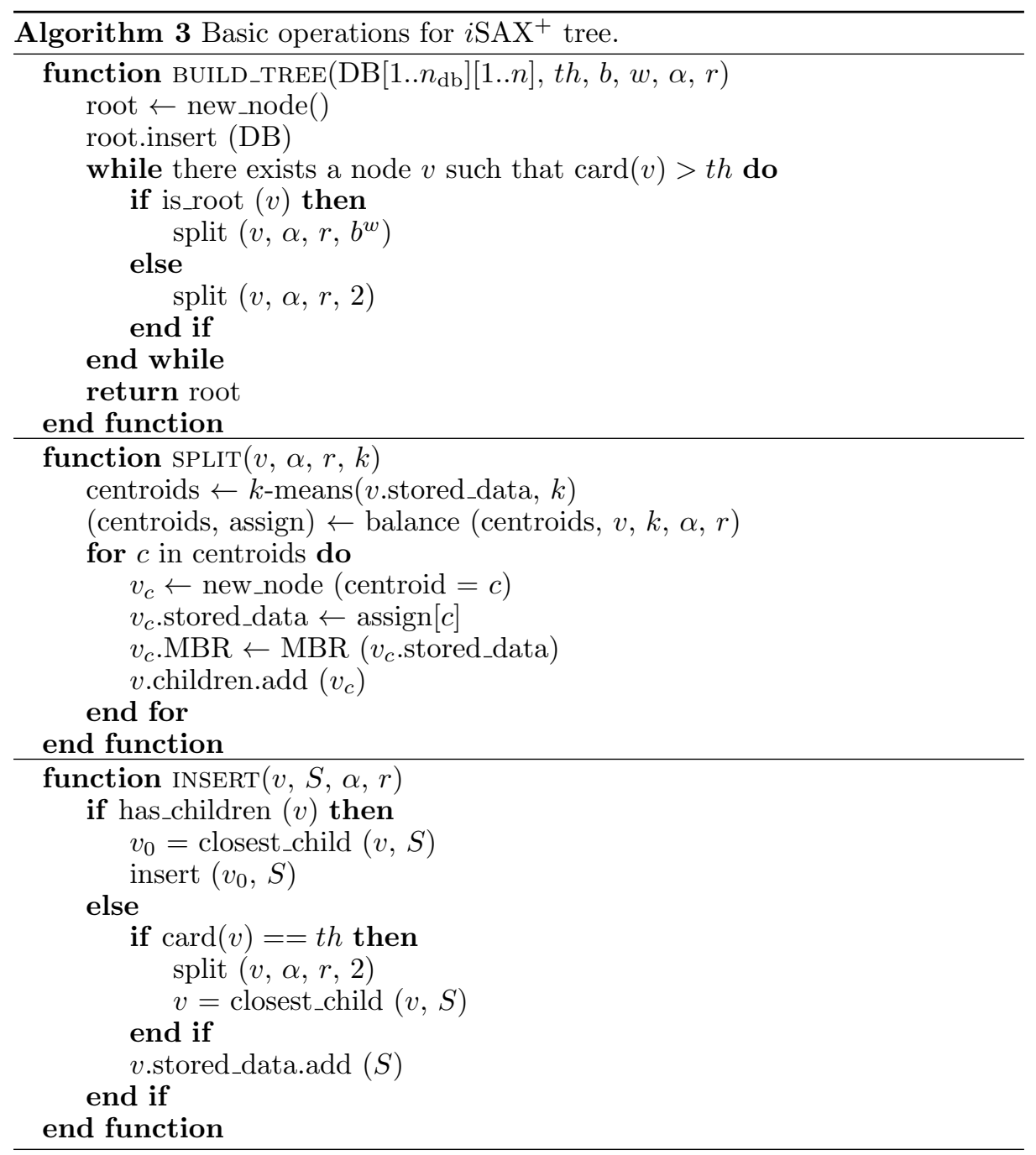

The experiments use two datasets. The first one, referred to as $\mathrm{DS}_{1}$, is precisely defined in Keogh and Ratanamahatana (2005): It is made of 32 time series, 50 subsequences of length 256 being randomly extracted from each dataset to build the test set. In order to learn our lookup table $\mathcal{C}$, we built our own learning set per time series using 100 randomly extracted subsequences of length 256 .

The second dataset, that we call $\mathrm{DS}_{2}$, is available at Keogh et al. (2006) and was used in several publications. It is made of 20 sub-datasets, each split in advance into a learning and a test set. When running experiments involving $\mathrm{DS}_{2}$ we naturally use these ready-made learning sets to build $\mathcal{C}$. Note that, these sub-datasets also provide classification labels for every sequence. When evaluating classification, we assign to the query the label of the most similar sequence returned by the search algorithm.

For datasets $\mathrm{DS}_{1}$ and $\mathrm{DS}_{2}$ and for both $\mathcal{A} \_$Keogh and $\mathcal{A} \_\mathrm{PAA}$, we report the 


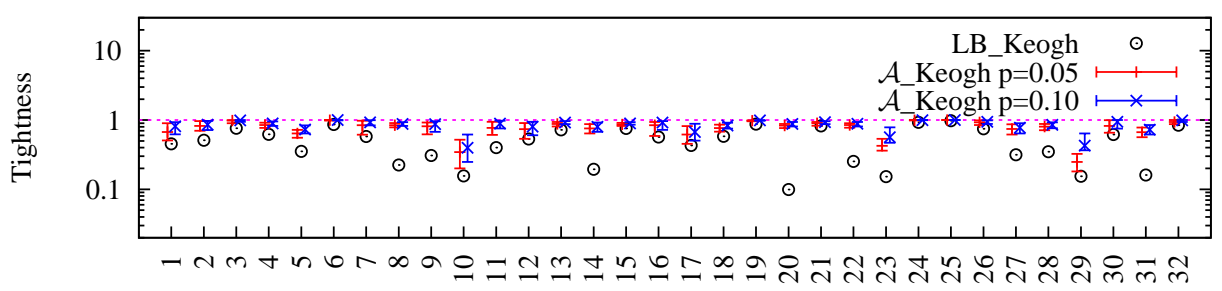

Sub-dataset

(a) $\mathrm{DS}_{1}$

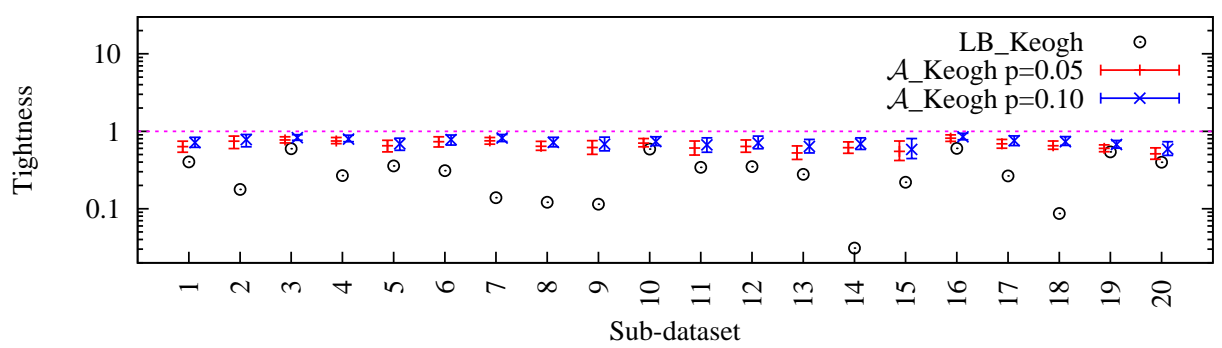

(b) $\mathrm{DS}_{2}$

Fig. 5. Median of tightness and its quartiles for $\mathcal{A}_{\_}$Keogh compared to the tightness of LB_Keogh.

first-nearest-neighbor (1-NN) retrieval rate, that is, the ratio of queries for which their true nearest neighbor (according to the DTW) is ranked first.

Note that, when necessary, estimator medians are reported together with their inter-quartile interval.

\subsection{Compared Tightness of Lower Bounds}

Figure 5 compares the tightness achieved by LB_Keogh and $\mathcal{A} \_$Keogh on datasets $\mathrm{DS}_{1}$ and $\mathrm{DS}_{2}$. It can be seen that the observed tightness for LB_Keogh varies significantly between series: For example, while it is tight for series \#3 or \#10 with $\mathrm{DS}_{2}$, it is very loose for series $\# 8$ or $\# 13$. In contrast, $\mathcal{A} \_$Keogh is overall much closer to 1 for both datasets. Note that, when $p=0.05$ or $p=0.1$, the improved tightness is achieved with high precision, as shown by the inter-quartile intervals that do not cross the $100 \%$ tightness limit for any dataset. As predicted, setting higher values for $p$ leads to higher achieved tightness. It is important to see that the results are more stable when using large learning datasets as it is the case of $\mathrm{DS}_{2}$, where inter-quartile intervals are so small that they can hardly be seen on figures.

\subsection{Similarity of Candidate Lists}

Algorithm 1 sorts all sequences from the database according to the value of $\mathcal{A} \_$Keogh. That order depends on the value given to $p$ and might might possibly differ from the one that is determined when sequences are ordered according to their LB_Keogh value. Moreover, with $\mathcal{A} \_$Keogh, when $p>0$, the break instruc- 


\begin{tabular}{|c|c|c|c|}
\hline Dataset & Method & $\begin{array}{l}\text { Median } \\
\text { of } \rho\end{array}$ & $\begin{array}{c}\text { Quartiles } \\
\text { of } \rho\end{array}$ \\
\hline \multirow{6}{*}{$\mathrm{DS}_{1}$} & LB_Keogh & 0.966 & {$[0.874 ; 0.996]$} \\
\hline & $\mathcal{A} \_$Keogh $p=0.05$ & 0.967 & {$[0.865 ; 0.997]$} \\
\hline & $\mathcal{A} \_$Keogh $p=0.1$ & 0.967 & {$[0.861 ; 0.997]$} \\
\hline & LB_PAA & 0.917 & $0.501 ; 0.991]$ \\
\hline & $\mathcal{A}$ A_PAA $p=0.05$ & 0.927 & {$[0.653 ; 0.993]$} \\
\hline & $\mathcal{A} \_$PAA $p=0.1$ & 0.926 & {$[0.653 ; 0.993]$} \\
\hline \multirow{6}{*}{$\mathrm{DS}_{2}$} & LB_Keogh & 0.914 & {$[0.820 ; 0.982]$} \\
\hline & $\mathcal{A} \_$Keogh $p=0.05$ & 0.914 & {$[0.819 ; 0.982]$} \\
\hline & $\mathcal{A} \_\operatorname{Keogh} p=0.1$ & 0.915 & {$[0.819 ; 0.982]$} \\
\hline & LB_PAA & 0.614 & $\mid 0.310 ; 0.875]$ \\
\hline & $\mathcal{A} \_$PAA $p=0.05$ & 0.661 & {$[0.194 ; 0.874]$} \\
\hline & $\mathcal{A} \_$PAA $p=0.1$ & 0.663 & {$[0.181 ; 0.867]$} \\
\hline
\end{tabular}

Table 2. Spearman correlation coefficient. Here, the median of $\rho$ values is computed for all sub-datasets and the corresponding quartiles are reported.

tion can be fired before identifying the best match, causing a false dismissal. It is therefore key to check if the order according to which sequences are sorted along their lower bound is somehow similar when considering DTW and LB_Keogh on the one hand or DTW and $\mathcal{A} \_$Keogh on the other. If the latter order is quite similar, then it is likely that the best match will also be returned when using $\mathcal{A} \_$Keogh.

The Spearman correlation coefficient $\rho$ is a widely used metric for assessing the similarity of two ordered lists. The closer to $1 \rho$ is, the more similar the lists are. It is defined as:

$$
\rho=1-\frac{6 \sum_{i=1}^{n_{\mathrm{db}}}\left(r_{i}^{1}-r_{i}^{2}\right)^{2}}{n_{\mathrm{db}}\left(n_{\mathrm{db}}^{2}-1\right)},
$$

where $r_{i}^{1}$ and $r_{i}^{2}$ are the ranks of sequence $i$ in both lists.

Table 2 gives the median values for $\rho$ computed on datasets $\mathrm{DS}_{1}$ and $\mathrm{DS}_{2}$. In this experiment, the set of $r_{i}^{1}$ has been produced by ordering the $n_{\mathrm{db}}$ sequences along their DTW value; $r_{i}^{2}$ is either LB_Keogh, $\mathcal{A} \_K e o g h$, LB_PAA or $\mathcal{A} \_P A A$. In all cases, the values for $\rho$ do not vary much when $p$ ranges from 0 to 0.1 . Note also the large overlap between inter-quartile intervals suggests the observed differences for $\rho$ between the three versions of $\mathcal{A} \_$Keogh (resp. $\mathcal{A} \_$PAA) are not significant. Finally, it is interesting to notice that $\mathcal{A} \_$PAA has higher $\rho$ values than LB_PAA. In other words, when a lower bound is far from tight, it can be useful to grasp information from the upper bound so as to generate ordered candidate lists that are closer to the ones that DTW would get.

Another important point is that the observed $\rho$ values are much smaller for LB_PAA (resp. $\mathcal{A} \_$PAA) than they are for LB_Keogh (resp. $\mathcal{A} \_$Keogh). This implies that smaller values should be used for $p$ when trying to approximate LB_PAA as each DTW overestimation is more likely to induce a false dismissal.

\subsection{Retrieval performances}

We used $\mathrm{DS}_{2}$ to evaluate the performances of our proposed approximate lower bounds in terms of retrieval performances as well as computational cost. Re- 


\begin{tabular}{ccccc}
\hline Method & $p$ & Cost & $1-\mathrm{NN}$ & Classification \\
\hline LB_Keogh & - & 1.000 & 1.000 & 0.918 \\
\hline \multirow{3}{*}{ A_Keogh } & 0.01 & 0.165 & 0.715 & 0.913 \\
& 0.10 & 0.062 & 0.410 & 0.879 \\
\hline \hline LB_PAA & - & 1.000 & 1.000 & 0.915 \\
\hline \multirow{2}{*}{ A_PAA } & 0.001 & 0.770 & 0.942 & 0.918 \\
& 0.01 & 0.370 & 0.603 & 0.906 \\
& 0.132 & 0.243 & 0.861 \\
\hline
\end{tabular}

Table 3. Compared accuracy and computational cost of $\mathcal{A} \_$Keogh and $\mathcal{A} \_$PAA. In all cases, the cost of the exact lower bound is used as a reference.

\begin{tabular}{cccccc}
\hline & Dataset & $T$ & Cost & $1-\mathrm{NN}$ & $\begin{array}{c}\text { Classi- } \\
\text { fication }\end{array}$ \\
\hline 1 & 50words & 0.402 & 0.437 & 0.802 & 0.765 \\
2 & Adiac & 0.177 & 0.182 & 0.673 & 0.519 \\
3 & Beef & 0.581 & 0.526 & 1.000 & 0.500 \\
4 & CBF & 0.268 & 0.236 & 0.953 & 0.994 \\
5 & Coffee & 0.357 & 0.372 & 0.929 & 0.821 \\
6 & ECG200 & 0.309 & 0.335 & 0.970 & 0.850 \\
7 & FISH & 0.138 & 0.354 & 0.874 & 0.851 \\
8 & FaceAll & 0.121 & 0.234 & 0.679 & 0.766 \\
9 & FaceFour & 0.114 & 0.728 & 0.966 & 0.841 \\
10 & Gun_Point & 0.589 & 0.428 & 0.967 & 0.920 \\
11 & Lighting2 & 0.342 & 0.599 & 0.984 & 0.852 \\
12 & Lighting7 & 0.349 & 0.566 & 0.945 & 0.808 \\
13 & OSULeaf & 0.277 & 0.211 & 0.839 & 0.612 \\
14 & OliveOil & 0.030 & 0.698 & 0.867 & 0.867 \\
15 & SwedishLeaf & 0.220 & 0.382 & 0.782 & 0.789 \\
16 & Trace & 0.600 & 0.597 & 0.890 & 0.990 \\
17 & Two_Patterns & 0.264 & 0.036 & 0.463 & 1.000 \\
18 & synthetic_control & 0.086 & 0.551 & 0.860 & 0.987 \\
19 & wafer & 0.541 & 0.294 & 0.748 & 0.986 \\
20 & yoga & 0.398 & 0.342 & 0.861 & 0.839 \\
\hline
\end{tabular}

Table 4. Per-dataset accuracy and computational cost. The cost of LB_Keogh is used as a reference and $\mathcal{A} \_$Keogh is used with $p$ set to 0.01 .

trieval is expressed by both the 1-NN retrieval rate and the correct classification rate. The computational cost is expressed as a ratio of the number of DTW computations induced by the approximate lower bound to the number of DTW computations induced by its corresponding exact lower bound. Note that, this cost does not include our off-line learning step, as it aims at reflecting on-line query cost.

Table 3 presents these results for $\mathcal{A} \_$Keogh and $\mathcal{A} \_$PAA. The first thing to notice is that in all cases, the computational cost decreases considerably even for small values of $p$. Moreover, as previously suggested, small values of $p$ should be used when considering LB_PAA to reduce the likelihood of false dismissals. The classification rate drops much slower than does the 1-NN retrieval rate, showing that when the true nearest neighbor is not ranked first, another sequence of the same class is, which is key to performance, quality-wise. For more detailed 


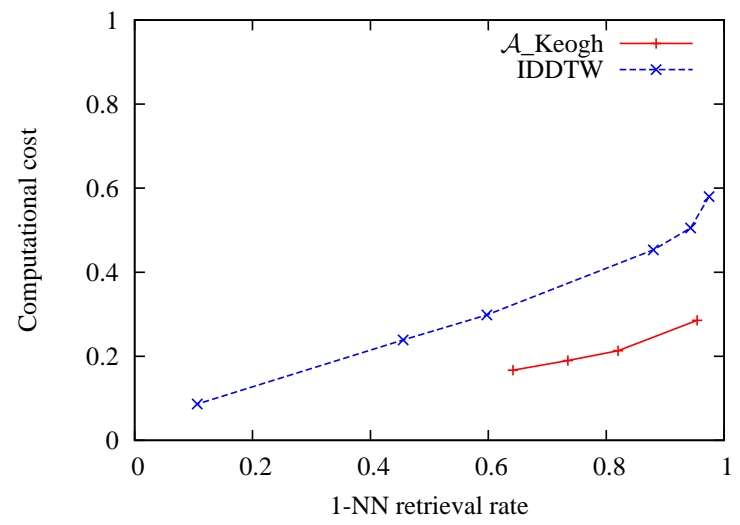

Fig. 6. Comparison of IDDTW and $\mathcal{A} \_$Keogh in terms of cost versus accuracy. The cost is defined as the number of elementary operations involved in the search process and accuracy is expressed in terms of $1-\mathrm{NN}$ retrieval rate.

information, refer to Table 4 that gives the cost versus accuracy trade-off for all sub-datasets in $\mathrm{DS}_{2}$.

\subsection{Comparison with IDDTW}

Both IDDTW and $\mathcal{A} \_$Keogh approaches rely on modeling the expected error that is induced by approximation. We therefore compare both approaches in terms of accuracy and computational cost, using $\mathrm{DS}_{1}$. Here, the computational cost is determined slightly differently from what was done previously, as IDDTW is an iterative process running DTW computations at different scales. We therefore define this cost as the total number of elementary distance computations that are needed for searching the database. For example, if a database of $n_{\mathrm{db}}$ sequences of length $n$ was searched using DTW with no temporal constraint, the cost would be $n_{\mathrm{db}} \times n^{2}$, since $n_{\mathrm{db}}$ DTW would be evaluated, each of which would use $n^{2}$ distance computations. This amount is then divided by the complexity of the reference method that is a full scan of the database using DTW constrained to a Sakoe-Chiba band.

Figure 6 shows that $\mathcal{A} \_$Keogh offers great improvement over IDDTW, as comparable retrieval performances can be obtained at a cost divided by two. This can be explained by the fact that each IDDTW comparison between the query and a candidate sequence uses several estimations to infer how likely that candidate is the best match. Running such estimations multiple times (IDDTW) introduces more errors than what a single test would do ( $\mathcal{A} \_$Keogh). That phenomenon, a well-known problem in statistics, explains why relying on $\mathcal{A} \_$Keogh better performs than using IDDTW.

\section{5. $i \mathrm{SAX}$}

In this section, we compare the performance of $\mathcal{A} \_i$ SAX and $\mathcal{A} \_i$ SAX_Approx with the performance of the original version of $i$ SAX2.0. We have built two indexing trees, one for learning and one for testing. The learning tree is required 


\begin{tabular}{cccccc}
\hline$p$ & Method & Cost & $1-\mathrm{NN}$ & $10-\mathrm{NN}$ & $50-\mathrm{NN}$ \\
\hline- & iSAX_MinDist & 1.0000 & 1.00 & 1.00 & 1.00 \\
- & iSAX_Approx & 0.0095 & 0.12 & 0.59 & 0.92 \\
\hline 0.001 & $\mathcal{A} \_$iSAX & 0.0722 & 0.39 & 0.84 & 0.99 \\
0.010 & $\mathcal{A} \_$SAX & 0.0324 & 0.18 & 0.77 & 0.99 \\
0.050 & $\mathcal{A} \_i$ SAX & 0.0034 & 0.03 & 0.26 & 0.77 \\
\hline 0.001 & $\mathcal{A} \_i$ SAX_Approx & 0.0014 & 0.12 & 0.57 & 0.92 \\
0.010 & $\mathcal{A} \_$iSAX_Approx & 0.0003 & 0.05 & 0.49 & 0.90 \\
0.050 & $\mathcal{A} \_i$ SAX_Approx & 0.0001 & 0.02 & 0.17 & 0.58 \\
\hline
\end{tabular}

Table 5. Retrieval performance, together with the ratio of DTW computations (denoted as cost), for $i \mathrm{SAX}$.

to determine the parameters needed by $\mathcal{A}_{-} i \mathrm{SAX}$ and $\mathcal{A} \_i \mathrm{SAX} \_\mathrm{Approx}$. Then, the learned parameters are used to construct the other tree, subsequently used for searching. Each tree stores 100,000 sequences that are random walks of length 256. Each node of the tree can store up to 1,000 sequences. The trees are built using the $i \mathrm{SAX} 2.0$ algorithm to ensure better balance in the leaves cardinality. Two sets of 1,000 random walk sequences are used as queries: one for learning and the other one for testing.

A groundtruth is computed in terms of DTW, as for previous experiments. Together with the 1-NN retrieval rate, we also report $10-\mathrm{NN}$ (resp. 50-NN) retrieval rate that is the ratio of returned nearest neighbors that are among the true 10 (resp. 50) nearest neighbors of the query point. $i$ SAX_MinDist is used as a reference in terms of cost.

We measured average query times of around 10s for iSAX_MinDist while approximate methods could perform several orders of magnitude faster: Querying the index took on average $100 \mathrm{~ms}$ for $i \mathrm{SAX} \_A p p r o x, 300 \mathrm{~ms}$ for $\mathcal{A} \_i \mathrm{SAX}$ with $p=0.01$ and $10 \mathrm{~ms}$ for $\mathcal{A}_{-} i \mathrm{SAX}$ _Approx with $p=0.01$. Note that, these timings are coherent with the cost expressed here (that is related to the number of DTW computations), which is why we will keep presenting this cost in the following tables.

Results presented in Table 5 show that $\mathcal{A} \_i$ SAX outperforms $i$ SAX_MinDist and that $\mathcal{A} \_i$ SAX_Approx outperforms $i$ SAX_Approx. For example, when $p=$ 0.001 , $i$ SAX_Approx runs more than 6 times more DTW computations than what $\mathcal{A} \_i$ SAX_Approx does while achieving similar retrieval rates (this refers to raws $\# 2$ and $\# 6$ of the table). It is interesting to observe the retrieval $10-\mathrm{NN}$ (resp. $50-\mathrm{NN}$ ) rates given by columns 5 and 6 . The rates are excellent while the computation cost is significantly reduced.

\section{6. $i \mathrm{SAX}^{+}$}

We compare here the performance of $i \mathrm{SAX} 2.0$ and $i \mathrm{SAX}^{+}$that build their index tree using two different approaches $\left(i \mathrm{SAX}^{+}\right.$uses $k$-means). We first ran a set of experiments using the very same datasets as the ones presented above, i.e., that are made with normally distributed random walks. It is crucial to note sequences built that are particularly adapted to $i \mathrm{SAX} 2.0$. As we did previously, we set the parameters for $i \mathrm{SAX}^{+}$using a first set of sequences. These parameters are then used in the experiments below. 


\begin{tabular}{ccc}
\hline \multirow{2}{*}{ Tree } & \multicolumn{2}{c}{ Imbalance factor $\gamma$} \\
\cline { 2 - 3 } & Test tree & Learning tree \\
\hline iSAX 2.0 & 1.926 & 1.927 \\
$i$ SAX $^{+}$ & 1.11 & 1.10 \\
\hline
\end{tabular}

Table 6. Compared balance of $i \mathrm{SAX}$ and $i \mathrm{SAX}^{+}$trees.

\begin{tabular}{|c|c|c|c|c|c|}
\hline$p$ & Search type & Cost & $1-\mathrm{NN}$ & $10-\mathrm{NN}$ & $50-\mathrm{NN}$ \\
\hline - & $i$ SAX_MinDist & 1.000 & 1.00 & 1.00 & 1.00 \\
\hline - & $i \mathrm{SAX}^{+}{ }_{\text {_MinDist }}$ & 1.7997 & 1.00 & 1.00 & 1.00 \\
\hline - & $i \mathrm{SAX}^{+} \_\mathrm{Approx}$ & 0.0141 & 0.12 & 0.47 & 0.81 \\
\hline 0.001 & $\mathcal{A}_{-} i \mathrm{SAX}^{+}$ & 0.6756 & 0.89 & 1.00 & 1.00 \\
\hline 0.010 & $\mathcal{A}_{-} i \mathrm{SAX}^{+}$ & 0.0400 & 0.46 & 0.99 & 1.00 \\
\hline 0.050 & $\mathcal{A} \__{\_} \mathrm{SAX}^{+}$ & 0.0004 & 0.05 & 0.37 & 0.88 \\
\hline 0.001 & $\mathcal{A}_{-} i \mathrm{SAX}^{+}{ }_{-} \mathrm{Approx}$ & 0.0022 & 0.11 & 0.47 & 0.80 \\
\hline 0.010 & $\mathcal{A} \_i \mathrm{SAX}^{+}$_Approx & 0.0004 & 0.11 & 0.34 & 0.77 \\
\hline 0.050 & $\mathcal{A}_{-} i \mathrm{SAX}^{+}{ }_{-} \mathrm{Approx}$ & 0.0001 & 0.02 & 0.15 & 0.47 \\
\hline
\end{tabular}

Table 7. Compared performances for approximate search using $i \mathrm{SAX}^{+}$and $i \mathrm{SAX} 2.0$. Sequences used here are drawn from normal distribution.

Both $i \mathrm{SAX} 2.0$ and $i \mathrm{SAX}^{+}$try as much as possible to determine their quantization intervals to get roughly equiprobable symbols. They use different mechanisms, however. To check the effectiveness of these mechanisms, we measured the imbalance of the final intervals determined on the one hand by $i$ SAX2.0 and on the other after having ran the cluster balancing phase that follows the completion of the $k$-means in the case of $i \mathrm{SAX}^{+}$. Table 6 shows the ratio between the less and the more probable symbols from the trees build by $i \mathrm{SAX} 2.0$ and $i \mathrm{SAX}^{+}$when using the learning or the test tree. This table shows the $i \mathrm{SAX}^{+}$ balancing phase achieves a much better balance of symbols probabilities than what iSAX2.0 does. This is a very nice result.

We now turn to the retrieval results when using $i \mathrm{SAX} 2.0$ or $i \mathrm{SAX}^{+}$for searching. These results are given in Table 7. $i \mathrm{SAX} 2.0$ and $i \mathrm{SAX}^{+}$show comparable retrieval performance when $p=0.001$-slightly lower in the case of 1-NN and identical otherwise. The retrieval cost is, however, much lower.

\begin{tabular}{|c|c|c|c|c|c|}
\hline$p$ & Search type & Cost & $1-\mathrm{NN}$ & $10-\mathrm{NN}$ & $50-\mathrm{NN}$ \\
\hline- & $i$ SAX_MinDist & 1.000 & 1.00 & 1.00 & $\overline{1.00}$ \\
\hline - & $i$ SAX_Approx & 0.0129 & 0.20 & 0.81 & 0.95 \\
\hline - & $i \mathrm{SAX}^{+}$_MinDist & 1.9314 & 1.00 & 1.00 & 1.00 \\
\hline- & $i \mathrm{SAX}^{+}{ }_{-}$Approx & 0.0159 & 0.14 & 0.53 & 0.76 \\
\hline 0.001 & $\mathcal{A} \_i \mathrm{SAX}$ & 0.2363 & 0.68 & 0.96 & 1.00 \\
\hline 0.001 & $\mathcal{A}_{2} i \mathrm{SAX}^{+}$ & 0.2914 & 0.95 & 1.00 & 1.00 \\
\hline 0.010 & $\mathcal{A}_{\_} i \mathrm{SAX}^{+}$ & 0.0125 & 0.24 & 1.00 & 1.00 \\
\hline
\end{tabular}

Table 8. Compared performances for approximate search using $i \mathrm{SAX}^{+}$and $i \mathrm{SAX} 2.0$ : the case of non-normal features 


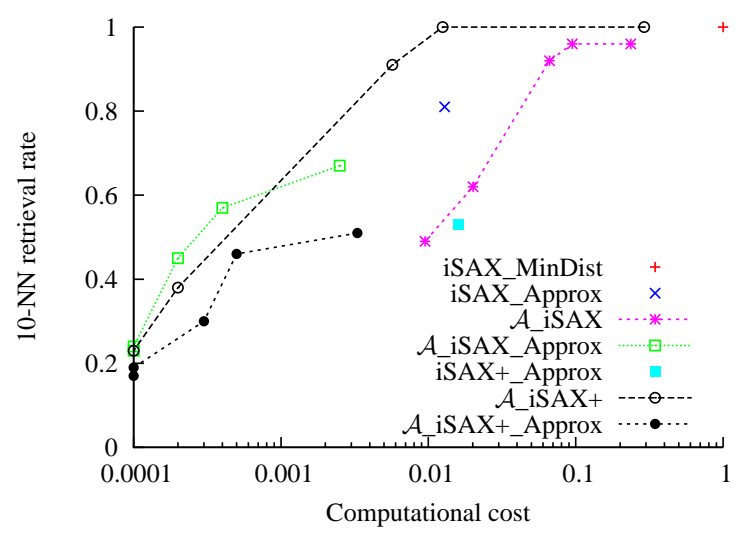

Fig. 7. Comparison of several indexing methods in the case of non-normal features.

One claim made in this paper is that $i \mathrm{SAX}^{+}$better performs than $i \mathrm{SAX} 2.0$ when non-normally distributed sequences are used. We therefore ran experiments involving random walks using a Beta distribution with parameters $\alpha=\beta=0.5$. There are 100,000 such walks in the database, each has a length of 256 and 1,000 other such walks have been created for querying the system. Table 8 presents the retrieval performances and the computing costs for $i \mathrm{SAX}^{+}$and $i \mathrm{SAX} 2.0$ when using these sequences. $\mathcal{A}_{\_} i \mathrm{SAX}^{+}$is much faster than $i \mathrm{SAX}$ MinDist and iSAX_Approx while achieving better recall compared to the latter.

Comparing Tables 7 and 8 enlighten the benefit of relying on a method that does not make use of any assumption on the distribution of the data in space. Figure 7 give more comprehensive results for this non-normal feature setup. It shows that the best trade-offs in this case are obtained either by $\mathcal{A}_{-} i \mathrm{SAX}$ A Approx or $\mathcal{A}_{\_} i \mathrm{SAX}^{+}$, depending on the computational cost one can afford.

\subsection{A case study: indexing DNA datasets}

As shown in Camerra et al. (2010), indexing full genomes using time series representations enables to link chromosomes between species that share common ancestors. For example, Rhesus macaques and humans have a common ancestor that lived 25 million years ago, which means their genomes share common attributes. Yet, the mapping between their chromosomes is not straightforward. One possible way to find such a mapping is to turn DNA sequences into time series and use related indexing techniques.

Here, we use the method proposed in Camerra et al. (2010), that is, each DNA symbol is changed into a numerical value equal to the one of the previous element in the sequence plus a value that depends on the symbol. Obtained sequences are then downsampled by a factor of 25 in order to reduce noise. We finally use a sliding window of length 400 and step 10 so as to extract subsequences. We use the $i$ SAX2.0 algorithm with parameters $w=10, b=2$, th $=1,000$ to index the whole Rhesus macaque genome (that is made of 10, 194, 500 such subsequences). The obtained index is queried using 300 sequences randomly picked from each human chromosome and their transposed versions. As in Camerra et al. (2010), we only consider longest chromosomes for the mapping, and for each of these 
chromosomes, we keep track of the 10 nearest neighbors among all retrieved neighbors.

This case study is typical of problems for which exact methods would be really long to run, while approximate approaches can provide useful information. This in fact motivated the use for $i$ SAX_Approx rather than $i$ SAX_MinDist in Camerra et al. (2010). For this reason, we chose to use a high value for our parameter $p$, that is $p=0.1$, so that low query times could be achieved. We present correspondence matrices in Fig. 8 by coloring each cell according to the amount of these nearest neighbors for the considered pair: the darker the cell, the more neighbors. Query times reached by $\mathcal{A}_{\_} i \mathrm{SAX}$ _Approx are significantly lower than the ones of $i$ SAX_Approx, while obtained correspondence matrices are visually similar. Note that, in this case, performing exact search would take around 100days (estimation based on the query times measured for a few of the 12,000 queries).

\section{Conclusion}

Reducing the cost of computing the DTW similarity measure between sequences is crucial to many applications. For some applications, indexing is not appropriate. Their performance, however, can be improved by using approximate lower bounding distance functions as described in this paper. The accuracy of these functions can range from rough results with possible false dismissals returned extremely fast to exact results returned more slowly-the users can set this accuracy versus speed trade-off. For other applications, indexing is mandatory, for example to cope with scale. In this case, these approximate lower bounding distance functions can also improve the performance of retrievals, as demonstrated here. Furthermore, this paper also shows that relying on a balanced $k$-means quantification step significantly improves the behavior of the very popular $i \mathrm{SAX}$ indexing scheme. Future work includes the design of more robust indicators than the s-DTW estimate. This includes the proposition of specific lower bounds dedicated to the match of rapidly varying sequences, for which LB_Keogh lacks tightness. We will also investigate the specific case of sequences of high-dimensional features, in which relying on assumptions on the distribution of the data is unaffordable. Finally, for many applications, the aim is not to match entire sequences, but rather spot similar subsequences inside long sequences. In this case, novel indexing approaches have to be developed to allow for approximate matching of subparts of time series.

\section{References}

Aach, J. and Church, G. (2001), 'Aligning gene expression time series with time warping algorithms', Bioinformatics $\mathbf{1 7}(6), 495$.

Camerra, A., Palpanas, T., Shieh, J. and Keogh, E. J. (2010), iSAX 2.0: Indexing and mining one billion time series, in 'Proceedings of the IEEE International Conference on Data Mining'.

Chu, S., Keogh, E., Hart, D., Pazzani, M. et al. (2002), Iterative deepening dynamic time warping for time series, in 'Proceedings of the SIAM International Conference on Data Mining'.

Faloutsos, C., Ranganathan, M. and Manolopoulos, Y. (1994), Fast subsequence matching in time-series databases, in 'Proceedings of the ACM SIGMOD Conference on Management Of Data'. 


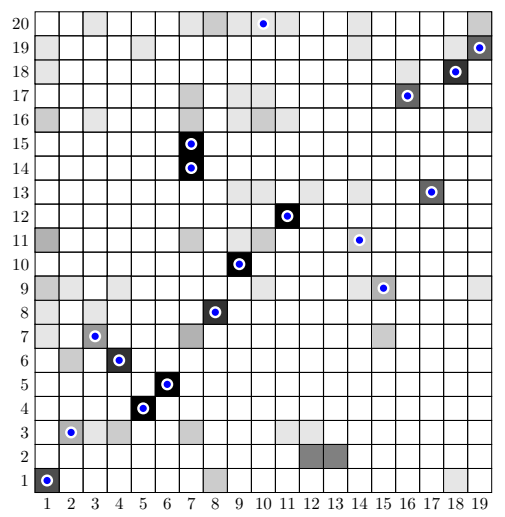

(a) iSAX_Approx (Query time: $5 \mathrm{~h}$ )

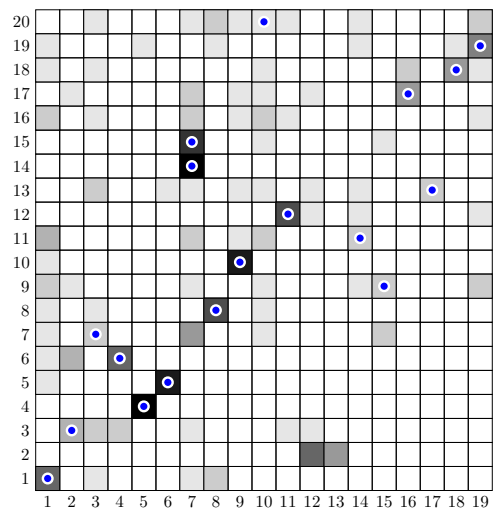

(b) $\mathcal{A} \_i$ SAX_Approx (Query time: $3 \mathrm{~h}$ )

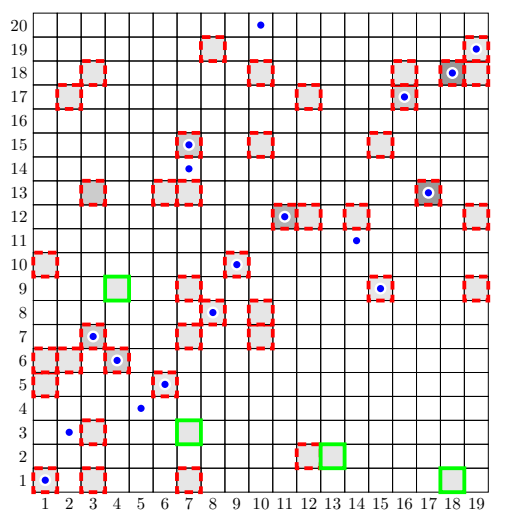

(c) Difference between both

Fig. 8. Correspondence matrices between human and Rhesus macaque genomes. Y-axis corresponds to human chromosomes, while $\mathrm{X}$-axis represents Rhesus macaque ones. Blue dots correspond to ground-truth matches. In (b), $p$ parameter is set to $10 \%$. Difference map is given to evaluate the similarity between both matrices, where white cells indicate perfectly similar results while black cells stand for opposite results. Cells marked with solid green line are those for which our algorithm is better while cells marked with dashed red lines are the ones for which $i$ SAX_Approx performs better. Other cells all have equal values for both techniques. Best viewed in color.

Gavrila, D. and Davis, L. (1995), Towards 3-d model-based tracking and recognition of human movement: a multi-view approach, in 'International workshop on automatic face-and gesture-recognition', pp. 272-277.

Itakura, F. (1975), 'Minimum prediction residual principle applied to speech recognition', IEEE Transactions on Acoustics, Speech and Signal Processing 23(1), 67-72.

Kashyap, S. and Karras, P. (2011), Scalable knn search on vertically stored time series, in 'Proceedings of the ACM SIGKDD Conference on Knowledge Discovery and Data Mining', ACM, pp. 1334-1342.

Keogh, E. and Ratanamahatana, C. (2005), 'Exact indexing of dynamic time warping', Knowledge and Information Systems 7(3), 358-386.

Keogh, E., Xi, X., Wei, L. and Ratanamahatana, C. A. (2006), 'The ucr time series classification/clustering homepage: www.cs.ucr.edu/ eamonn/time_series_data/'.

Lin, J., Keogh, E., Wei, L. and Lonardi, S. (2007), 'Experiencing SAX: a novel symbolic representation of time series', Data Mining and Knowledge Discovery 15(2), 107-144. 
Munich, M. and Perona, P. (1999), Continuous dynamic time warping for translation-invariant curve alignment with applications to signature verification, in 'Proceedings of the IEEE International Conference on Computer Vision', Vol. 1, pp. 108-115.

Nistér, D. and Stewénius, H. (2006), Scalable recognition with a vocabulary tree, in 'Proceedings of the IEEE Conference on Computer Vision and Pattern Recognition', pp. 2161-2168.

Paulevé, L., Jégou, H. and Amsaleg, L. (2010), 'Locality sensitive hashing: A comparison of hash function types and querying mechanisms', Pattern Recognition Letters 31(11), 1348 $-1358$.

Sakoe, H. and Chiba, S. (1978), 'Dynamic programming algorithm optimization for spoken word recognition', IEEE Transactions on Acoustics, Speech, and Signal Processing 26, 4349.

Shieh, J. and Keogh, E. (2008), iSAX: Indexing and mining terabyte sized time series, in 'Proceedings of the ACM SIGKDD Conference on Knowledge Discovery and Data Mining'.

Sivic, J. and Zisserman, A. (2003), Video Google: A text retrieval approach to object matching in videos, in 'Proceedings of the IEEE International Conference on Computer Vision', pp. $1470-1477$.

Tavenard, R., Jégou, H. and Amsaleg, L. (2011), Balancing clusters to reduce response time variability in large scale image search, in 'Proceedings of the IEEE Workshop on ContentBased Multimedia Indexing'.

Vlachos, M., Hadjieleftheriou, M., Gunopulos, D. and Keogh, E. J. (2003), Indexing multidimensional time-series with support for multiple distance measures, in 'Proceedings of the ACM SIGKDD Conference on Knowledge Discovery and Data Mining', pp. 216-225.

Yi, B., Jagadish, H. V. and Faloutsos, C. (1998), Efficient retrieval of similar time sequences under time warping, in 'Proceedings of the IEEE International Conference on Data Engineering'. 


\section{Appendices}

\section{A. Proofs and mathematical definitions for upper bounds}

We prove here that UB_Keogh is upper bounding DTW when the latter is restricted to a Sakoe-Chiba band. We also introduce upper bounds related to LB_PAA and $i$ SAX_MinDist for which we omit the proofs as they follow the exact same principles.

Definition 1. Let UB_Keogh be:

$\mathrm{UB} \_\operatorname{Keogh}(Q, C)$

$$
=\sum_{i=1}^{n} \begin{cases}\left(c_{i}-L_{i}\right) & \text { if } c_{i}>U_{i} \\ \left(U_{i}-c_{i}\right) & \text { if } c_{i}<L_{i} \\ \max \left(U_{i}-c_{i}, c_{i}-L_{i}\right) & \text { otherwise }\end{cases}
$$

Lemma 1. For any two sequences $Q$ and $C$ of length $n$, the following inequality stands:

$$
L_{1}(Q, C) \geq \operatorname{DTW}(Q, C)
$$

where the considered DTW is constrained to a Sakoe-Chiba band of width $r$.

Proof. Let $Q$ and $C$ be two sequences of length $n$. Manhattan distance $L_{1}$ corresponds to the alignment that follows the diagonal path. Hence, this distance is associated with one of the possible paths considered by the DTW algorithm and is therefore greater than the cost of the minimal path, that is the value returned by DTW, which concludes the proof for Lemma 1.

Proposition 1. For any two sequences $Q$ and $C$ of length $n$, the following inequality stands:

$$
\mathrm{UB} \_\operatorname{Keogh}(Q, C) \geq \operatorname{DTW}(Q, C)
$$

where the considered DTW is constrained to a Sakoe-Chiba band of width $r$.

Proof. It is important to notice that each term in the sum that occurs in the definition of UB_Keogh is related to exactly one term in the computation of the $L_{1}$ distance. The only difference is that for UB_Keogh, the $i$-th term corresponds to the distance between the $i$-th point in the candidate sequence and its furthest corresponding point in the envelope of the query, while for $L_{1}$ the same term is equal to the distance between the $i$-th point in the candidate sequence and one of its possible corresponding points in the envelope of the query. The latter distance is then, by definition, smaller than the former, and the following inequality is then straightforward, coming from Lemma 1:

$\mathrm{UB} \_\operatorname{Keogh}(Q, C) \geq L_{1}(Q, C) \geq \operatorname{DTW}(Q, C)$. 
Definition 2. Let us define UB_PAA as:

$$
\begin{aligned}
& \text { UB_PAA }(Q, C) \\
& \begin{array}{ll}
=\frac{n}{N} \cdot\left(\sum_{i=1}^{N} \max \left(\hat{U}_{i}-\bar{c}_{i}, \overline{c_{i}}-\hat{L}_{i}\right)\right) \\
+\frac{n}{N} \cdot(\max (C)-\min (C)) .
\end{array}
\end{aligned}
$$

Lemma 2. For any two sequences $Q$ and $C$ of length $n$, the following inequality stands:

$$
\operatorname{UB} \_P A A(Q, C) \geq \text { UB_Keogh }(Q, C) \text {. }
$$

Proposition 2. For any two sequences $Q$ and $C$ of length $n$, the following inequality stands:

$$
\operatorname{UB} \_P A A(Q, C) \geq \operatorname{DTW}(Q, C)
$$

where the considered DTW is constrained to a Sakoe-Chiba band of width $r$.

Proof. It is straightforward that proving Lemma 2 is sufficient to prove, using Proposition 1, that Proposition 2 holds.

Let $Q$ and $C$ be two sequences of length $n$ and $W$ be the minimum cost path used for DTW computation with a Sakoe-Chiba band of with $r$ between $Q$ and $C$. So as to prove:

$$
\sum_{i=1}^{n} \max \left(U_{i}-c_{i}, c_{i}-L_{i}\right) \leq \frac{n}{N} \cdot\left(\sum_{i=1}^{N} \max \left(\hat{U}_{i}-\bar{c}_{i}, \bar{c}_{i}-\hat{L}_{i}\right)+\max (C)-\min (C)\right)
$$

it is sufficient to prove that, for all $i \in\{1, \ldots, N\}$ :

$$
\sum_{j=\frac{n}{N}(i-1)+1}^{\frac{n}{N} i} \max \left(U_{j}-c_{j}, c_{j}-L_{j}\right) \leq \frac{n}{N} \cdot\left(\max \left(\hat{U}_{i}-\bar{c}_{i}, \bar{c}_{i}-\hat{L}_{i}\right)+(\max (C)-\min (C))\right.
$$

Let $i \in\{1, \ldots, N\}$. If we denote, for all $j \in\left\{\frac{n}{N}(i-1)+1, \ldots, \frac{n}{N} i\right\}, c_{j}=\overline{c_{i}}+\Delta c_{j}$, 
we get :

$$
\begin{aligned}
& \sum_{j=\frac{n}{N}(i-1)+1}^{\frac{n}{N} i} \max \left(U_{j}-c_{j}, c_{j}-L_{j}\right) \\
= & \sum_{j=\frac{n}{N}(i-1)+1}^{\frac{n}{N} i} \max \left(U_{j}-\left(\overline{c_{i}}+\Delta c_{j}\right), \overline{c_{i}}+\Delta c_{j}-L_{j}\right) \\
\leq & \sum_{j=\frac{n}{N}(i-1)+1}^{\frac{n}{N} i} \max \left(\hat{U}_{i}-\left(\overline{c_{i}}+\Delta c_{j}\right), \bar{c}_{i}+\Delta c_{j}-\hat{L}_{i}\right) \\
\leq & \sum_{j=\frac{n}{N}(i-1)+1}^{\frac{n}{N} i} \max \left(\hat{U}_{i}-\bar{c}_{i}, \bar{c}_{i}-\hat{L}_{i}\right)+\left|\Delta c_{j}\right| \\
\leq & \frac{n}{N}\left(\max \left(\hat{U}_{i}-\bar{c}_{i}, \bar{c}_{i}-\hat{L}_{i}\right)+\max (C)-\min (C)\right)
\end{aligned}
$$

which concludes the proof.

Definition 3. Let us define iSAX_MaxDist as:

$$
i \operatorname{SAX} \_\operatorname{MaxDist}(Q, R)
$$

$$
=\sqrt{\frac{n}{N} \sum_{i=1}^{N} X_{i}}
$$

where

$$
\forall i \leq N, X_{i}= \begin{cases}\left(\overline{q_{i}}-B_{i}\right)^{2} & \text { if } \overline{q_{i}}>H_{i} \\ \left(H_{i}-\bar{q}_{i}\right)^{2} & \text { if } \overline{q_{i}}<B_{i} \\ \max \left(H_{i}-\bar{q}_{i}, \bar{q}_{i}-B_{i}\right)^{2} & \text { otherwise }\end{cases}
$$

\section{B. Balancing $k$-means}

When $k=2$, balancing $k$-means does not require any iterative process as proposed in (Tavenard et al., 2011). It is possible to derive elevation $h$ that the most populated clusters' centroid will get in order for both clusters to finally get equal populations without resorting to any iterative process.

Let us assume, without loss of generality, that $k$-means produced two centroids $\mathbf{C}_{1}$ and $\mathbf{C}_{2}$ and that cluster $C_{1}$ is more populated than $C_{2}$. Using notations introduced in Fig. 9, intersection between the line $\left(\mathbf{C}_{\mathbf{1}}, \mathbf{C}_{\mathbf{2}}\right)$ and the boundary between classes $C_{1}$ and $C_{2}$ is then $\mathbf{C}_{\mathbf{0}}$, middle of the line segment $\left[\mathbf{C}_{\mathbf{1}}, \mathbf{C}_{\mathbf{2}}\right]$. We aim at evaluating elevation $h$ such that this point moves to $\mathbf{C}_{\mathbf{0}}^{\prime}$ that is the median of projected data points. It is straightforward that if one builds a new boundary that is parallel to the original one and passes through $\mathbf{C}_{\mathbf{0}}^{\prime}$, both clusters will be equally populated. After solving the related system of equations, one gets:

$$
h=\sqrt{2\left(x_{2}-x_{1}\right)\left(\frac{x_{1}+x_{2}}{2}-x_{0}^{\prime}\right)},
$$

where $x_{1}$ and $x_{2}$ are known from the $k$-means and $x_{0}^{\prime}$ is the median of projected data points. 


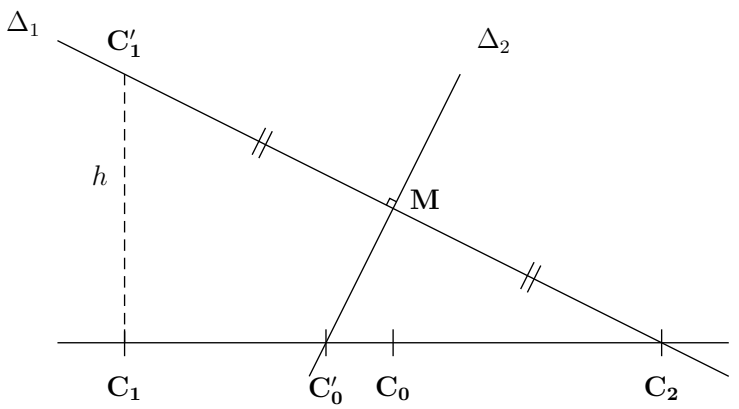

Fig. 9. Balancing $k$-means for the $k=2$ case.

\section{Author Biographies}

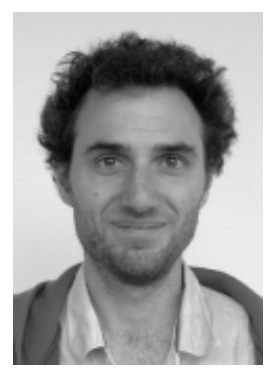

Romain Tavenard received a M.Sc. degree from École Centrale de Lyon (Lyon, France) in 2006. He then spent one year with CWI lab (Amsterdam, The Netherlands). He received a Ph.D. from Université de Rennes 1 (Rennes, France) in 2011. From 2011 to 2013, he worked at Idiap Research Institute (Martigny, Switzerland) as a post-doctoral researcher and then joined Université de Rennes 2 (Rennes, France) as Assistant professor. His main research interests include data mining and indexing of time series, with application to multimedia or geoscience data.

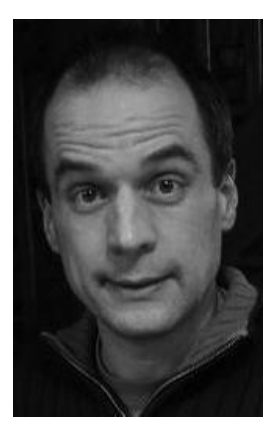

Laurent Amsaleg received his Ph.D. in June 1995 from the University of Paris 6, France. He worked on relational and object-oriented databases, garbage collection, micro-kernels and single-level stores. He then spent 18 months in the Database group of the University of Maryland (MD, USA), designing flexible database query execution strategies (Query Scrambling). Subsequently, he received a full-time research position at CNRS in France and joined the IRISA lab in Rennes. His research primarily focuses on content based multimedia retrieval, which include the design of multidimensional indexing techniques. Recently, Laurent turned his focus to security problems, breaking the recognition power of content based image retrieval systems and challenging privacy of multimedia contents.

Correspondence and offprint requests to: Romain Tavenard. Université de Rennes 2, Rennes, France. Email: romain.tavenard@univ-rennes2.fr 